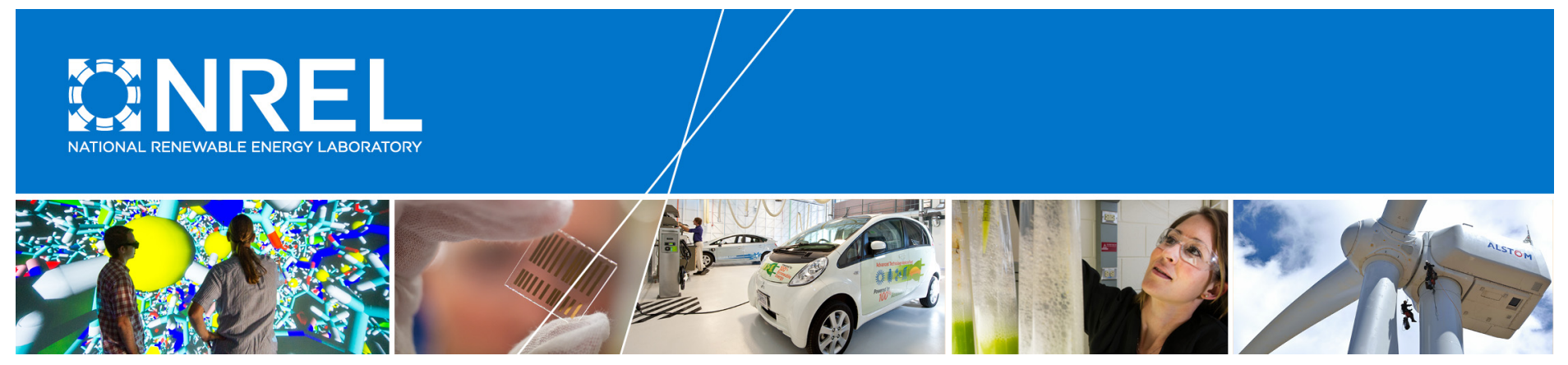

\title{
2017 Marine Hydrokinetic Instrumentation Workshop Report
}

Frederick Driscoll

National Renewable Energy Laboratory

Erik Mauer

U.S. Department of Energy

Jeff Rieks

Allegheny Science and Technologies

NREL is a national laboratory of the U.S. Department of Energy Office of Energy Efficiency \& Renewable Energy

Operated by the Alliance for Sustainable Energy, LLC

This report is available at no cost from the National Renewable Energy Laboratory (NREL) at www.nrel.gov/publications.

Technical Report

NREL/TP-5000-70591

March 2018

Contract No. DE-AC36-08G028308 


\section{Marine Hydrokinetic Instrumentation Workshop Report}

Frederick Driscoll

National Renewable Energy Laboratory

Erik Mauer

U.S. Department of Energy

Jeff Rieks

Allegheny Science and Technologies

\section{Suggested Citation}

Driscoll, Rick, Erik Mauer, and Jeff Rieks. 2018. 2017 MHK Instrumentation Workshop Report. Golden, CO: National Renewable Energy Laboratory. NREL/TP-5000-70591. https://www.nrel.gov/docs/fy18osti/70591.
National Renewable Energy Laboratory 15013 Denver West Parkway

Golden, CO 80401

303-275-3000 • www.nrel.gov
NREL is a national laboratory of the U.S. Department of Energy Office of Energy Efficiency \& Renewable Energy Operated by the Alliance for Sustainable Energy, LLC

This report is available at no cost from the National Renewable Energy Laboratory (NREL) at www.nrel.gov/publications.

\section{Technical Report}

NREL/TP-5000-70591

March 2018

Contract No. DE-AC36-08GO28308 


\title{
NOTICE
}

This report was prepared as an account of work sponsored by an agency of the United States government. Neither the United States government nor any agency thereof, nor any of their employees, makes any warranty, express or implied, or assumes any legal liability or responsibility for the accuracy, completeness, or usefulness of any information, apparatus, product, or process disclosed, or represents that its use would not infringe privately owned rights. Reference herein to any specific commercial product, process, or service by trade name, trademark, manufacturer, or otherwise does not necessarily constitute or imply its endorsement, recommendation, or favoring by the United States government or any agency thereof. The views and opinions of authors expressed herein do not necessarily state or reflect those of the United States government or any agency thereof.

This report is available at no cost from the National Renewable Energy Laboratory (NREL) at www.nrel.gov/publications.

Available electronically at SciTech Connect http:/www.osti.gov/scitech

Available for a processing fee to U.S. Department of Energy and its contractors, in paper, from:

\author{
U.S. Department of Energy \\ Office of Scientific and Technical Information \\ P.O. Box 62 \\ Oak Ridge, TN 37831-0062 \\ OSTI http://www.osti.gov \\ Phone: 865.576.8401 \\ Fax: 865.576.5728 \\ Email: reports@osti.gov
}

Available for sale to the public, in paper, from:

\author{
U.S. Department of Commerce \\ National Technical Information Service \\ 5301 Shawnee Road \\ Alexandria, VA 22312 \\ NTIS http://www.ntis.gov \\ Phone: 800.553 .6847 or 703.605 .6000 \\ Fax: 703.605.6900 \\ Email: orders@ntis.gov
}




\section{Acknowledgments}

Thank you to all the invited speakers and participants in the third Marine Hydrokinetic Instrumentation Workshop. Their insights and willingness to share their experiences about developing marine energy technologies helped make the workshop a success.

We thank the session leads, Terry Lettenmaier, Levi Kilcher, and Eric Nelson, for facilitating discussions and documenting the findings. In addition, we thank Dr. Manhar Dhanak and Florida Atlantic University (FAU) for hosting the workshop at FAU's SeaTech campus, a beautiful waterfront facility in Dania Beach, Florida. We thank Arielle Cardinal for organizing the meeting and facilitating communication. Finally, we thank Giorgio Bacelli, Ryan Coe, Jon Keller, Genevra Harker-Klimes, Terry Lettenmaier, Rolf Lueck, Pedro Lomonaco, Vince Neary, Brian Polagye, Robert Raye, Jim Thomson, Jeroen van Dam, and Rick Williams for providing valuable feedback on various sections of the report.

We also gratefully acknowledge the financial support from the U.S. Department of Energy's Water Power Technologies Office, which enabled us to organize, conduct, and report on the outcomes of this workshop. 


\section{Nomenclature or List of Acronyms}

6DOF

ADCP

ADP

AEP

AIS

CEC

COTS

DAS

DOE

DOF

EMI

GPS

IEA-OES

IEC

IMU

IP

LCOE

MEC

MHK

MHKDR

MOIS

MRU

NREL

O\&M

ONR

PNNL

PTO

QA

R\&D

RDT\&E

RF

TPL

TRL

USCG

WEC six degrees of freedom

acoustic Doppler current profiler

acoustic Doppler profiler

annual energy production

Automatic Identification Systems

current energy converter (tidal, river, open-ocean)

commercial off the shelf

data acquisition system

U.S. Department of Energy

degree of freedom

electro-magnetic interference

Global Positioning System

International Energy Agency - Ocean Energy

Systems

International Electrotechnical Commission

inertial measurement unit

intellectual property

levelized cost of energy

marine energy converter

marine hydrokinetic

Marine and Hydrokinetic Data Repository

Modular Ocean Instrumentation System

motion reference unity

National Renewable Energy Laboratory

operation and maintenance

Office of Naval Research

Pacific Northwest National Laboratory

power take-off

quality assurance

research and development

research, development, test, and evaluation

radio frequency

technology performance level

technology readiness level

United States Coast Guard

wave energy converter 


\section{Executive Summary}

The third Marine Hydrokinetic Instrumentation Workshop was held at Florida Atlantic University's Sea Tech Campus in Dania Beach, Florida, from February 28 to March 1, 2017. The workshop brought together 37 experts in marine energy measurement, testing, and technology development to present and discuss the instrumentation and data-processing needs of the marine energy industry. The goals of the meeting were to:

- Better understand the state of instrumentation, measurement, and data-processing capabilities for testing, assessment, monitoring, and operation of marine energy converters (MECs)

- Identify gaps (problems, deficiencies, or lack of capabilities) in marine hydrokinetic (MHK) measurement and data-processing technology, assess the impact of those gaps, and prioritize the gaps for solutions

- Define pathways for developing solutions to the gaps

- Present progress made to address gaps identified at previous workshops.

The workshop was comprised of a plenary session followed by two focused breakout sessions. The half-day plenary session reviewed findings from prior instrumentation workshops, presented research activities that aim to fill previously identified gaps, and had industry experts present the state of the marine energy measurement technologies. The bulk of the workshop was spent in the breakout sessions where the workshop participants split into three groups 1) wave energy converter (WEC) field testing and operation, 2) current energy converter (CEC) field testing and operation, and 3) laboratory testing (WEC and CEC). The first session defined and ranked gaps in measurement technology and data processing. The second session defined ways to develop measurement and data-processing technologies to fill the gaps.

The first section of this report provides the background for the workshop, reviews the objectives, and details the structure. The body of this report details the findings from the workshop in terms of gaps in existing technology, capabilities, and infrastructure. The findings also include descriptions of the benefits of closing the gaps and potential solutions to those gaps. A final section describes the common themes that emerged in several of the findings.

The workshop identified 28 primary findings in the areas of

- Information dissemination and data processing,

- Standards, guidelines, and recommended practices

- Enhanced measurement capabilities

- New measurement and testing capabilities 
Four cross-cutting themes emerged from many of the findings:

- Limited knowledge transfer: a wealth of experience, knowhow, and tools have been generated from laboratory and field testing, yet much of this knowledge is not disseminated or is hard to find. There is a need to disseminate knowledge so the MHK industry can avoid repeating mistakes, minimize duplicate efforts, and leverage the experience of others to help accelerate technology development and reduce costs while not compromising intellectual property.

- High cost of measurement: marine grade instruments can be expensive and strain tight test budgets; often leading to a tradeoff between the breadth and duration of a test and the number and quality of measurements. By adopting mechanisms to increase instrument availability, share instrument costs between projects, increase the measurement capabilities of existing instruments, adapt instruments for MHK application, share knowledge of instrument use, and standardize measurements, measurement costs can be reduced.

- Better measurement capabilities at low technology readiness levels (TRLs): measurement capabilities for smaller-scale models (1:10 and smaller) typically used at low TRLs are inadequate and sensors either do not exist, are too expensive, or adversely impact device response. For technologies to advance at smaller TRLs, new measurement technologies are needed to enable accurate and affordable measurements.

- Open-source tools for unified data processing and analysis: data processing and analysis is typically performed on a project-by-project basis using custom code with unique processing and visualization methods. By encouraging sharing of vetted data reduction, processing, QA, and visualizations code and by adopting standard methods, the MHK industry would be able to accelerate the analysis and increase the credibility of test results.

The gaps, impacts of the gaps, benefits of closing the gaps, and solutions to the gaps contained within this report were synthesized from the preworkshop survey and input from workshop participants. The findings are intended to be informative and used by government bodies, industry and the research community to help advance MHK testing and measurement - they do not necessarily represent the U.S. Department of Energy (DOE) Water Power Technologies Office (WPTO) views or program objectives. 


\section{Table of Contents}

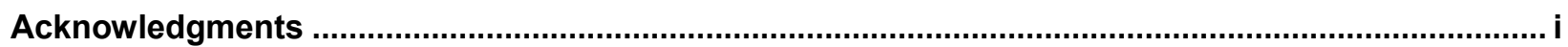

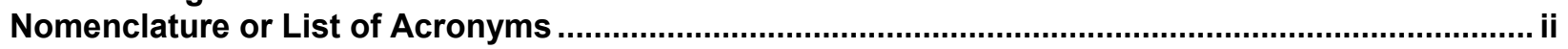

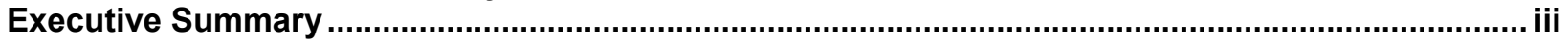

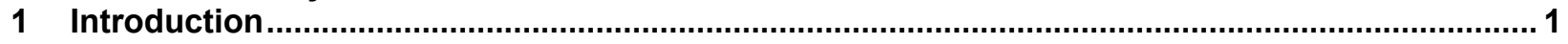

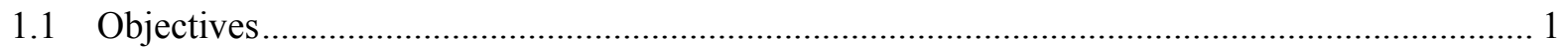

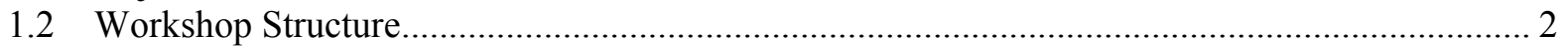

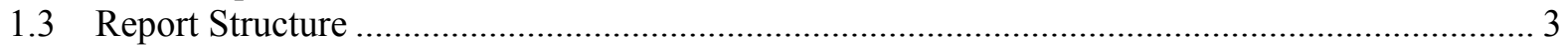

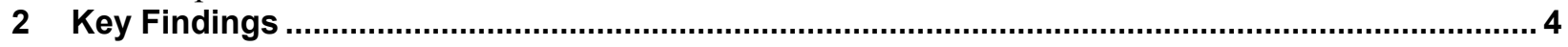

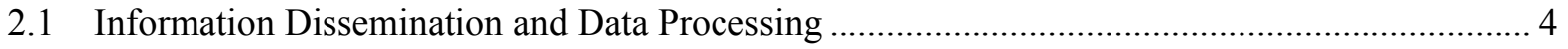

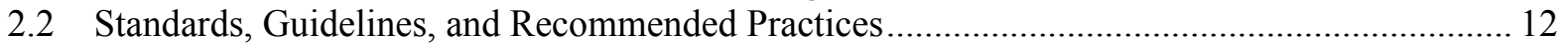

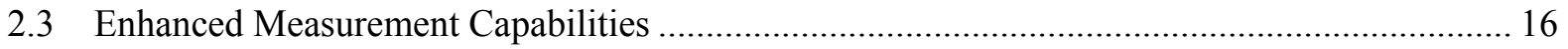

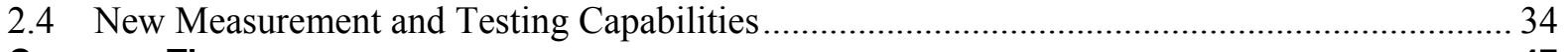

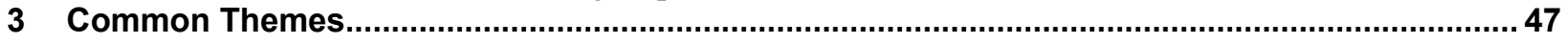

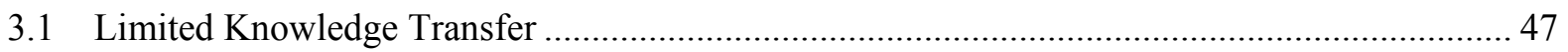

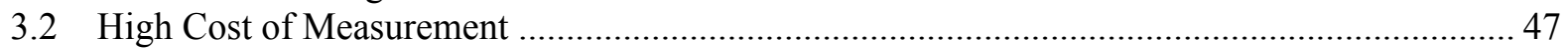

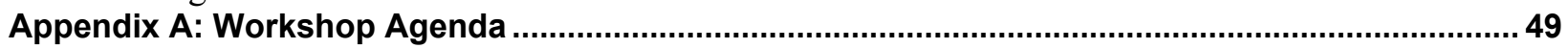

Appendix B: Pre-Workshop Survey Questions....................................................................... 52

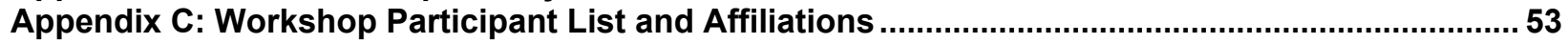




\section{Introduction}

This report presents the findings of the third workshop in the area of marine hydrokinetic (MHK) sensors and instrumentation sponsored by the U.S. Department of Energy (DOE) Water Power Technologies Office (WPTO). The workshop was held at Florida Atlantic University's Sea Tech Campus in Dania Beach, Florida, from February 28 to March 1, 2017 and brought together 37 experts in marine energy measurement, testing, and technology development.

The prior two workshops focused on all areas of measurement and modeling for both MHK and offshore wind with a goal of sharing state-of-the-art equipment and methods, field testing experiences, and lessons learned. Environmental monitoring was not in the scope at a prior workshop hosted by the Pacific Northwest National Laboratory (PNNL) - the Instrumentation for Monitoring Around Marine Renewable Energy Converters Workshop. ${ }^{1}$ This third workshop instead focused on identifying measurement and data-processing needs, determining gaps in measurement capabilities, and defining solution pathways for those gaps, as relevant for laboratory and field testing and operation of MHK technologies. The workshop theme was:

Instrumentation and data processing to advance marine hydrokinetic energy in the laboratory and in the field: How do we get the information we need?

The focused technical workshop included 37 participants from industry, national laboratories, and academia. The size of the workshop was intentionally kept small to facilitate open and indepth discussions about various subjects relevant to the MHK industry. Participants were technical experts in the fields of instrumentation, monitoring, testing, and data analysis. They had hands-on roles in the development or use of instrumentation in support of offshore wind and MHK technology research, development, testing and evaluation or aligned and relevant fields.

\subsection{Objectives}

The workshop focused on marine grade instrumentation systems used for open-water site characterization, structural field testing, certification testing and verification, commissioning, operational monitoring, and controlled testing in a laboratory environment. The technology scope is offshore wind, wave, and current (tidal, river, and open-ocean) systems in water-based testing facilities and at open water sites.

The workshop had four objectives:

- To better understand the state of instrumentation, measurement, and data-processing capabilities for testing, assessment, monitoring, and operation of marine energy converters (MECs)

- To identify gaps (problems, deficiencies, or lack of capabilities) in MHK measurement and data-processing technology, assess the impact of those gaps, and prioritize the gaps for solutions

- To define pathways to developing solutions to the gaps

- To present progress filling gaps identified at previous workshops.

${ }^{1}$ http://www.pnnl.gov/main/publications/external/technical_reports/PNNL-23100.pdf. 


\subsection{Workshop Structure}

The two-day workshop was comprised of a general half-day plenary session, focused breakout sessions with smaller groups (see agenda in Appendix A), and reporting from each breakout session. This structure was chosen to facilitate in-depth discussions and exchanges of information that would not have been possible in a presentation to a large audience.

A pre-workshop survey was sent out in advance of the workshop to query participants and the wider community about the state-of-the-art, gaps, and research and development (R\&D) challenges related to MHK measurement systems, instruments, and sensors. The results were compiled and presented at the opening of the workshop. The compilation was a resource document for the breakout groups and designed to help start and focus discussion. The survey consisted of four questions (see Survey in Appendix B).

The half-day opening plenary session consisted of three parts: 1) a welcome and workshop overview, 2) an overview of DOE WPTO-sponsored projects aimed at filling gaps that were identified at prior workshops, and 3) presentations by keynote speakers who are experts in MHK instrumentation, measurement, and data-processing fields. DOE welcomed the participants, provided the workshop background, highlighted the intended workshop outcomes, and overviewed the findings from other workshops. The National Renewable Energy Laboratory (NREL) provided an overview of the workshop, reviewed the agenda, and detailed participant roles and the intended outcomes.

The first set of presentations informed participants of the impacts of prior workshops by presenting DOE WPTO sponsored projects that had their genesis from previous workshop findings. A presentation was also given that provided an overview of the state-of-the-art of the MHK industry in Canada. The second set of presentations provided overviews of the state-ofthe-art in measurement technologies and testing projects. The last set of presentations set the stage for discussions in the breakout groups.

Prior to entering the breakout groups, NREL presented the findings from the pre-workshop survey. Each technical expert participated in one of the three breakout groups:

1. Wave energy converter (WEC) field testing and operation

2. Current energy converter (CEC) field testing and operation

3. Laboratory testing of WEC and CEC technologies.

Each breakout group had a session lead and was provided with an Excel spreadsheet to guide and document their findings. The goal of the first breakout session was to define and rank the gaps in measurement and data-processing technology. The gaps were defined in terms of 1) a description of the desired measurement, capability, or data set, 2) a description of the gap based on the current state-of-the-art, 3) the impact of the gap, 4) the benefits of filling the gap, and 5) a description of potential solutions. On the morning of the second day, the session lead reported the findings to the entire workshop for broader discussion. 
The second breakout session focused on brainstorming pathways to solution(s) to the measurement and data-processing gaps identified in the first breakout session. Participants documented the roles of industry, national laboratories, test facilities, and academia in the proposed development paths. The session leads reported the findings to the entire workshop for discussion in the afternoon on the final day.

Following the workshop, NREL compiled the findings from the breakout sessions and sent them out to the workshop participants and the larger MHK community to review, provide feedback, and prioritize the gaps in order of impact.

To the best of our knowledge, the findings in this document represent some of the current gaps that impede the development of MHK technologies. While not identified within the scope of this work, some solutions to these gaps may exist in whole or in part, and a community of practice has been developed based on feedback from similar workshops to provide a forum to share information and knowledge relevant to the larger MHK community.

Also note that the workshop findings were synthesized from the preworkshop survey and input from participants and they are intended to be informative and used by government bodies, industry and the research community to help advance MHK testing and measurement. The findings do not necessarily represent the U.S. Department of Energy (DOE) Water Power Technologies Office (WPTO) views or program objectives.

If you would like to share or discuss existing solutions to the gaps identified, please visit the MHK Community of Practice at https://www.nrel.gov/water/mhk-instrumentation-sensordatabase.html or contact the authors of this report.

\subsection{Report Structure}

The main body of this report is structured around the findings from the breakout groups. The findings from each breakout session are grouped in four themes:

- Information dissemination and data processing

- Standards, guidelines, and recommended practices

- Enhanced measurement capabilities

- New measurement and testing capabilities.

Each finding is presented as a separate narrative with the background and desired capability described first. Next, the gap in technology, infrastructure, or information is described along with identification of the impact of the gap on advancing MHK technology. Finally, the benefits of closing the gap and potential solutions are detailed. 


\section{Key Findings}

\subsection{Information Dissemination and Data Processing}

\subsubsection{MHK Instrumentation Database: Increased Capabilities and Awareness}

There is a large range of sensors and instruments produced by many different companies that can be used by the MHK community for both laboratory and field experiments, tests, and operational measurements. It can be very difficult to locate a sufficient sensor/instrument-let alone the best sensor/instrument for a given task. This occurs for a number of reasons, including: there are a large number of sensors/instruments with no all-inclusive search mechanism; there are no consensus metrics for sensor/instrument performance specification; the IP67/68 (International Protection water resistance ratings) may not be applicable to seawater; different sensors/instruments can work better or worse depending on the application; and deployment environment and marine grade sensors/instruments can require special installation/protection methods that may not be obvious at the time of selection. Configuring these sensors/instruments can also be challenging because knowledge of the sensor/instrument operation and physics is often needed to obtain the best performance.

An online database of sensors, instruments, and data acquisition hardware used for resource assessment, field and laboratory testing, and operation (monitoring of deployed MECs) of MHK technologies with user experiences and recommended uses/configurations would be useful to the MHK community. Such a database should be searchable for and/or include information on

1) Instrumentation cost

2) Summary measurement information and performance specs

3) Manufacturers

4) Configuration and setups

5) User experience

6) Links to manuals

7) Owners of instruments who are willing to loan or lease the instruments.

Ideally, the database should be open to users to upload new content and edit existing content. It should also be widely used by DOE awardees (and other communities) to capture, to the broadest extent possible, the knowledge and experience gained through DOE-funded activities and the overall MHK community.

Currently, the DOE MHK instrumentation database partially meets this need as it is a free community-developed database that houses an extensive list of MHK-related testing sensors and instrumentation. The database can be modified and expanded by registered users. In its current form, most information has been provided by DOE in its initial seeding. Limited information has been provided by the public. 
Gaps: The existing MHK Instrumentation Database (https:/www.nrel.gov/water/mhkinstrumentation-sensor-database.html) is a good start and has a reasonable number of instruments but needs more instruments in areas of environmental monitoring and sensors for underwater load measurements. It currently lacks user experiences and recommended settings, and other application-specific guidance is missing. Fields do not exist for instrumentation costs, measurement ranges, owners of instruments, and instrument performance, and no mechanism exists for feedback by users. Also, the existing population and structure leans more toward field testing than laboratory testing.

One of the more significant gaps is that, while useful, the existing database has not seen significant utilization and remains unknown to the larger MHK community.

Impact of Gaps: Without an instrumentation database that meets the full needs and satisfies the gaps above, the following can occur:

- Reduced measurements or data quality because the proper sensors are not always selected and/or configured correctly (both field and laboratory measurements)

- Increased costs and slower technology development resulting from replacement of sensors, repeated tests, and inadequate data for technology evaluation - data sets may be incomplete or data may not satisfy the project requirements

- Additional time taken to identify the correct instruments or selecting the incorrect instrument

- Increased costs because of a missed opportunity to leverage existing instruments via leasing/rental instead of purchasing such instruments

- Lack of capturing and sharing knowledge and experiences.

\section{Benefits of Closing the Gaps:}

- Shortening selection time and a higher likelihood of choosing the correct instrument which results in reduced testing time and costs, and yielding better data collection

- Increase measurement success and data quality because knowledge and experiences are shared

- Increase stakeholder engagement and database contributions

- Better meeting of user needs by modifying the database in a timely manner by adding a feedback button

- Reduce project costs by offering instrument sharing/loaning to users

- Improve design feedback and information to guide future iterations, leading to reduced time to commercialization and better device performance

- Yield better proof-of-concept evaluation for innovative designs at lower technology readiness levels (TRLs) through higher-quality data. 
Solutions: Update the functions and framework of the database to:

- Include a feedback button with a drop list of choices for the focus of the feedback (e.g., site functionality, issues) and a text field for detailed comments

- Add fields for:

○ Instrumentation cost

- Measurement range

O Owners of instruments

Instrument performance

- Modify structure or add structure and content to help meet the information needs for laboratory measurements

- Increase use and content population through increased awareness via contacting instrument manufacturers, outreach and publications at meetings and workshops (MHK and more broadly-related meetings), and capturing experiences gained through DOE WPTO funded activities.

\subsubsection{Open-Source Data Reduction, Conditioning, and Processing Code}

Programming and scripting languages such as MATLAB, Python, C, and R are commonly used in the MHK field for data reduction, conditioning, processing, manipulation, and visualization. The codes contain many built-in functions that allow users to develop powerful processing tools; however, they do not contain many of the functions that are needed by the MHK community (e.g., calculate wave energy flux based on the International Electrotechnical Commission (IEC)accepted method, plot a wave rose, determine a wave power matrix or CEC power curve, calculate the significant wave height from a spectrum). Thus, significant effort is often dedicated to developing processing codes and tools to support the needs of individual projects - much of this effort is redundant because there is significant overlap in the needed functionality. These codes do not typically represent a competitive advantage, yet they typically remain in-house with limited sharing internally and externally. For example, the national laboratories have developed analysis codes to support work on the Wave Energy Prize, validation of Wave Energy Converter SIMulator (WEC), reference model testing, and controls development. These codes are not available to the community or even shared between DOE national laboratories. While there are several public open-source libraries with some of the needed functions (such as oceanographic tools), programmers can invest substantial time finding them and then verifying that the codes are correct and/or meet the desired need.

Implementation of an online MHK data-processing code repository specific to marine energy with version control and search capabilities organized by function and language will be useful to help the MHK community. This would help the community to discover existing code that allows quicker and more consistent data processing - and perhaps establish common processing practices. Code should be divided up into specific functions that perform a single task, thus allowing users to pick functions they need without having to wade through large codes to cut out the needed capabilities. For specific cases, such as IEC analysis (e.g. a power curve) a complete code would be needed to make sure that the inputs and setting are correct and provides IEC compliant results - this will reduce variability and develop analysis capabilities bases on a 
consistent interpretation and application of the standards. The DOE national laboratories developed a suite of tools, such as crunch, mcrunch or GPP which were widely used within the wind industry and became commonly accepted analysis tools.

Codes/functions should all be well commented and use a standardized input/output model. In the repository, users would be able to search for and download functions to meet their needs, modify the code, and, if enhancements are added, upload the enhanced versions. Moderators should review code changes and release those that meet quality and performance requirements. This site should also contain a collection of pointers to other sites that have useful processing codes and tools to avoid duplication on the site.

Gaps: Code-sharing sites for processing and analyzing data-sharing sites exist in other fields (e.g., oceanography) and are widely used within those fields to help the community to more quickly process data. Currently, the Marine and Hydrokinetic Data Repository (MHKDR, https://www.nrel.gov/water/mhk-instrumentation-sensor-database.html) is the only site known to exist in the MHK community to share measurement information, and it does not have the functional capabilities needed to support code sharing (e.g., version control, searching, uploading/downloading). Open-source code-sharing capabilities have been developed for WECSim, but no online community tool is known to exist with sufficient functionality for sharing data processing and analysis code specific to marine energy.

\section{Impact of Gaps:}

- Higher costs and increased project duration because significant funding and time are invested in developing custom data-processing codes

- Divergent analysis and conclusions, non-conformity to accepted practices and standards, reduced credibility of findings, and lower investor confidence are some results of using custom codes developed on a project-by-project basis

- Higher probability of errors in code, resulting in errors - possible design flaws and failures.

Benefits of Closing the Gaps: Public open-source data-processing tools will help the MHK community produce credible results using consistent and vetted processing tools and techniques. Some benefits include:

- Lower cost and quicker data processing because needed code functions can be quickly found and integrated into a larger processing tool; because code developed by individual projects would be shared and leveraged by others

- High-quality figures and graphics that conform to commonly accepted layouts because plotting code would be available to, and enhanced by, the user community

- Lower probability of errors (higher confidence in the results) because code would be traceable to sources where code has been vetted by industry experts and other users

- Reduced redundancy as one set of code is used instead of everyone developing and validating their individual piece of code

- Increased confidence in the correctness of the test results by other stakeholders like banks and insurance companies because analysis is done using validated code. 
Solutions: Many online open-source code portals exist for oceanography and other fields but none that completely meet the needs for MHK data processing. Existing DOE code portals can be leveraged (i.e., WEC-Sim, https://wec-sim.github.io/WEC-Sim/) to share code for the MHK community, but they currently have insufficient functionality to meet the MHK data processing needs. The recommended solution steps are:

- Develop guidelines for code interfaces (if it does not exist) and for data reduction, conditioning, processing, manipulation, and visualization - consensus and vetted functions

- Develop a general DOE code-sharing portal (this could be done by leveraging an existing portal like the WEC-Sim portal), augment an aligned website such as the MHK Instrumentation Database (https://www.nrel.gov/water/mhk-instrumentation-sensordatabase.html) or develop a separate portal framework

- Linking existing MHK resources including code portals through the MHK community of practice.

- Encourage sharing of analysis software, perhaps through the U.S. International Energy Agency - Ocean Energy Systems (IEA OES) or other national/international programsspecifically, engage DOE national laboratories to upload their code, which should include specific functions (e.g., a function to calculate the wave energy flux using the method given in IEC 62600 - 100) and complete project codes

- Implement a database to share data-processing code on OpenEI and/or use DOE Code (https://www.osti.gov/doecode/)

- Identify and support code curators to establish initial content

- Develop guidelines for processing MHK data based on accepted practices and standards.

\subsubsection{Open-Source Code for Automated Data QA During Data Acquisition in the Field and Laboratory}

Quality assurance (QA) of field and laboratory test data and operational data of MECs is typically performed on a set-by-set basis during post processing, and live data streams are not usually monitored using automated codes to flag questionable data. When QA routines are developed, much like the data-processing code of the prior finding, significant effort is often dedicated to developing custom QA codes for individual projects - much of this effort is redundant because there is significant overlap in the needed functionality. Similarly, these codes do not represent a competitive advantage, yet they typically remain in-house with limited sharing internally and externally. The DOE national laboratories and many technology developers have created data QA codes for use in MHK and in aligned fields such as wind. For example, the national laboratories have developed QA codes to evaluate the Wave Energy Prize 1:20 scale data in near-real time. It would be useful to have a publicly available set of QA routines that have been vetted for which the MHK community can use to evaluate data as they are collected.

It would be useful to have experience and consensus-based guidance for QA of MHK data and a collection of automated processing tools that are able to screen and evaluate data as they are collected in the field/laboratory. Then questionable data could be flagged to alert data users of possible issues. These tools should also send real-time reports of erroneous data or sensor/instrumentation failures so that appropriate action can be taken in a timely manner. 
Ideally, the methods of QA data should be based on methods in other fields and are subject to peer review. QA code should be made available in common acquisition languages (e.g., LabView) and processing languages (e.g., Python, MATLAB).

Gaps: Considerable time is required to perform QA checks on large data sets - if they are performed at all. Often custom code is developed and direct human involvement is needed and applied on a set-by-set basis. QA of field data is typically limited to summary reports. Public, open-source, and MHK-relevant QA code that has been reviewed/vetted by subject matter experts is not known to exist and is not able to perform real-time data QA and reporting.

\section{Impact of Gaps:}

- Increased project duration and costs often result because QA systems are typically unique codes that are developed on a project-by-project basis.

- Time lags can occur between the first occurrence of a data issue and identification of this issue without real-time reporting. This could result in reduced test data (fewer channels) because data from bad sensors continue to be collected and issues are not identified and fixed during testing. Extended test duration can also result because more test time would be required to fill data gaps once an instrument/sensor/data acquisition system (DAS) issue has been fixed. Without data QA, there is decreased confidence in the data.

- False conclusions, bad designs being moved forward, or designs being moved in directions that have little or no benefit can result because erroneous data may be used when not performing QA.

\section{Benefits of Closing the Gaps:}

- Identifying problems/issues with measurements/sensors/hardware early, thereby allowing intervention at the earliest possible time, hence larger data sets, reduced test duration, and lower project costs

- Flagging data of questionable quality before public dissemination (e.g., data in the MHKDR could be flagged while still classified as "raw") to prevent use of such data

- Ensuring high-quality data and reducing testing development costs and timelines, thereby increasing knowledge used to guide future designs, which results in accelerated technology advancement.

Solutions: Automated data QA is common in other fields but has not been widely applied to the MHK field (e.g., Quality Assurance of Real-Time Oceanographic Data, QARTOD). Some capability exists to control data collection during specific activities, notify data users of data issues, and screen for specific data sets. To fill the gaps, leverage existing experience and code to perform the following steps:

- Develop and publish guidelines for QA of MHK data

- Develop open-source data screening/QA algorithms with real-time implementation in mind, vet those tools through users, and share those tools via an online code repository

- Develop event-detection methods using existing data sets

- Develop and apply machine learning (training and self-classification of data) to establish normal/abnormal data. 


\subsubsection{Open-Source Data Conversion Tools Based on Consensus File Formats}

Field and laboratory measurements are typically stored in file formats specific to their DAS and in a structure that is custom to the specific projects. Because file formats and data structure are often unique, custom routines need to be developed to load and parse each file format into common structures, such as those used by numerical simulation tools (e.g., to import sea-state data to drive the simulation). Standardizing test data file formats and structure, along with opensource tools that convert measurement outputs into a suitable format for model inputs would be helpful to the analysis and modeling community. It would help them quickly load data from different measurement sets without the need for custom code.

Gaps: No standardized data file format and structure exist and no open-source tools are known to exist that import or convert field/laboratory data so they can be used by numerical models such as WEC-Sim.

\section{Impact of Gaps:}

- Investment of significant time and project cost to convert custom data files to formats usable by numerical models and other analysis tools

- Occurrence of errors if the conversion and file format and structure, along with the import process, are not fully understood.

\section{Benefits of Closing the Gaps:}

- $\quad$ Reduced project time and budget

- Greater confidence in simulations results when based on imported data

- Easier and more numerical simulation validation.

\section{Solutions:}

- Develop standardized/generally agreed-upon data formats, structures, and variable naming conventions

- Create open-source tools for processing data from measurements to model input.

\subsubsection{Automated Analysis of Large Volumes of Data}

Large data sets are being created by the MHK industry through activities such as site characterization, resource assessment, environmental monitoring, testing, and device operation. These data include site conditions (wave velocity time series and wave spectra), device performance (power, mechanical loads, motion, and noise), and environmental monitoring (passive and active acoustics). These data sets can range in size up to many terabytes and may be distributed over many different files - in the case of a long-duration device test, tens of thousands of files can be created. Presently, processing is often done on segments of a data set using custom code or Excel spread sheets, often developed on a project-by-project basis. To efficiently mine these data sets for information that can help advance the MHK industry, opensource tools are needed that automatically QA, process, and analyze the high volumes of data to calculate accepted metrics (e.g., power curves/matrices), identify issues (e.g., power quality), and identify events (e.g., blade strike). A single software tool able to perform all data analysis and 
visualization task needs for the MHK community would be very difficult and expensive to create; however, tools that are designed for specific high-level tasks, such as ingesting data and filling in a power performance matrix or calculating fatigue cycles from large data sets collected from tests lasting more than a year, would be more feasible. Such tools would integrate lowerlevel software tools, as identified in the findings Open-Source Data Reduction, Conditioning, and Processing Code; Open-Source Code for Automated Data QA During Data Acquisition in the Field and Lab; and Open-Source Data Conversion Tools, into developing the higher-level tools. Also, to provide wide use with minimal customization, common sensors, file types/formats, and processing methods need to be agreed upon as identified in the finding Unified Sensing Technology, Measurement Methodology, Data Collection, and Data Processing. Tools should be developed for both environmental and engineering data.

Gaps: No open-source automated turnkey capability for processing and visualizing large volumes of data using consistent methods based on MHK guidelines, standards, and accepted practices are known to exist.

\section{Impact of Gaps:}

- Higher costs and long project durations because large efforts and time are invested in developing data-processing codes on a project-by-project basis, if at all

- Inconsistent processing between projects, possibly leading to inconsistent outcomes, because unique codes are used in different projects; higher probability of errors in code, resulting in erroneous results are also possible

- Lack of automated codes, which leads to data processing that is labor intensive and may miss key events; this can also create a lag time for information to be made available to help the project and/or be publicly disseminate.

\section{Benefits of Closing the Gaps:}

- Public tools that have been vetted by the community would help the MHK community produce credible results with a lower probability of errors because code would be consistent and techniques verified.

- A publicly available set of codes would reduce the need for unique, project-by-project development, thereby lowering project costs and yielding quicker data-processing timelines.

- Common data sets and data visualizations/graphics would be produced that can be used for cross-project data analysis and data mining-greatly expanding on what can be done with existing content models without increasing the burden on those submitting data. 


\section{Solutions:}

- Survey industry and test centers; review guidelines, standards, and accepted practices; and use field campaigns (environmental and technical) to determine what processing methods, data, and data products are needed by the MHK industry, certification bodies, and government agencies; utilize the information to develop an organized software framework that can meet the needs and is prioritized for development

- Survey test centers, companies, universities, and national laboratories to determine what code already exists and, when code is not yet in the public domain, incentivize for release

- Develop common data loading, reduction, QA, and processing code, expanded beyond what is done for the content model auto-population scripts

- Standardize MHK data repository metadata entry form, including data decoding information, so data can be easily and automatically ingested by analysis programs; this effort should attempt to automate metadata population to reduce the burden on submitters

- Cross-reference instruments between instrumentation database and the processing code so once an instrument is used, analysis routines would automatically be able to download and use appropriate code

- Pursue development of advanced methods for large-scale data processing, such as event detection (e.g., failures, animal strikes, and extreme events) and machine learning algorithms to organize data and determine normal versus abnormal.

\subsection{Standards, Guidelines, and Recommended Practices}

\subsubsection{Unified Testing Practices, Sensing Technology, Measurement Methodology, Data Collection, and Data Processing}

The MHK industry is still nascent, and accepted practices for field and laboratory measurements and data processing and analysis either do not exist or are at very early stages of development. At low TRLs, the IEC is developing laboratory and small-scale field testing standards for wave and current technologies. While these are not yet available, methods and techniques can be adopted from aligned fields such as naval architecture and aeronautics. At higher TRLs, IEC TC 114 technical specifications can be used, but many are still under development (such as mechanical loads) or have not started development. Many companies may also forego purchasing standards at their early stages of development to reduce costs or because they are unaware of their applicability. As a result, field and laboratory measurements and data-processing and analysis methods are chosen on a case-by-case/project-by-project basis and not on consensus standards and guidelines. Additionally, many sensors/instruments may be chosen for different reasons that include cost, availability, perceived importance, lack of knowledge, or because the limitations of sensors are unknown. As well, measurement and signal-conditioning specifications (e.g., sample rates, sensor locations, data filtering, and sensor response/sensitivity) can also be chosen on a project-specific basis. Decisions on what data to measure, the measurement specifications, and processing and analysis methods are often based on current needs, experience from prior projects, forecast needs, and cost. Thus, similar projects with similar technologies and testing goals can have significantly different sensors/instruments used and data in terms of quantities being measured. Because of the project-specific nature of measurements and data, the broader applicability of the data can be limited and cross-technology/cross-testing comparisons are 
difficult. Without foreknowledge of data needed to support later design iterations, measurements and analysis may only be chosen to support testing and evaluation of current technology; data may be insufficient to support future design activities.

It would be useful for the industry to have TRL-specific public guidance documents and standards that clearly define the types of measurements, types of sensors/instruments, where to make measurements, minimum measurement specifications by the type of test, how to conduct the test to produce correct and sufficient data, and how to process the results so they are credible and comparable. Such documents and standards would be intended to serve as information resources to help guide technical experts in instrumentation and data processing and should not be considered substitutes to domain expertise and experience.

Gaps: Unlike other industries, the MHK industry has not converged to a set of accepted sensors, instruments, or group of sensors and instruments for specific measurement categories and tests for the various TRL levels. At low TRLs, no guidelines or standards exist that provide measurement and analysis specifications for this early-stage pre-certification testing in the laboratory for loads, performance, and motion; through IEC TC 114, there is an effort to provide a solution. Also, because the cost and limited accessibility of the IEC publications under development, the community is not familiar with the content in the standards and/or is not applying the requirements of the standards for pre-commercial higher TRL testing.

\section{Impact of Gaps:}

- Sub-optimal data collection-limited data and poor-quality data, as well as data gaps (insufficient data to support evaluation and future development)

- Lack of common sensor/instrument used for specific measurements; lack of measurements in necessary locations (e.g., might miss critical strain measurements); and lack of common measurement specifications, which leads to limited data consistency between projects

- Lack of understanding between test/tank facility operators, developers, and other stakeholders.

Benefits of Closing the Gaps: Unified testing practices, sensing technology, measurement methodology, data collection, and data processing can lead to:

- Higher-quality and sufficient data that can support device evaluation, future design, and permitting because the methods and requirements are based on hands-on experiences and developed for the type of test and TRL - ultimately improved understanding and performance of MECs

- More straightforward development of common tools and processing techniques because common and agreed-to measurements, processing techniques, and testing practices exist 
- Selection of the best or most suitable sensors/instruments for the test and desired measurement

- Common data quality, data formats, processing tools, and metrics that allow straightforward cross-technology comparison

- Feedback to help advance standards and guidelines and a better understanding of requirements for field and laboratory test projects.

\section{Solutions:}

- Develop common and agreed-to measurements, processing techniques, and testing practices; as part of this effort, continue to develop TC 114 standards and testing guidelines that define different types of test and measurement by TRL/scale

- Ensure test facilities adopt common testing practices

- Ensure government-sponsored projects adhere to, as it makes sense and is practical, consensus testing standards and guidelines

- Develop procedures to calibrate and qualify instruments and sensors to meet agreed-upon requirements; then develop a list of accepted/qualified sensors/instruments for different tests

- Support research to determine the best placement of sensors that will minimize the number of measurements and fully capture all necessary information

- Review existing literature (including wind energy related standard in the IEC 61400 series, floating structures, oil \& gas, and other fields) to leverage existing efforts on conformity in measurement, processing, and data visualization

- Encourage and/or incentivize greater involvement by the MHK community in standards and guidelines development.

\subsubsection{AIS Standards for MEC On-Station and Off-Station/Private Aids to Navigation Standard}

Automatic Identification Systems (AISs) are common tools used to share position, track, and identify information between ocean-going vessels and shore stations. AISs are required navigation and safety equipment for larger vessels in U.S. and international waters. This technology could be adapted by the marine energy industry to provide real-time identification of marine traffic to MECs and as a safety mechanism to identify and locate MECs that are off station.

AIS codes are established through international standards bodies and implemented through national agencies. Under normal operating procedures, AISs are for vessels. It would be helpful to establish a standard for AIS codes for marine energy applications.

Gaps: Uniformly accepted international standards and United States Coast Guard (USCG) directives for marine energy applications do not exist. 


\section{Impact of Gaps:}

- Higher probability for vessel-MEC collision because MECs do not show up on shipborne AIS systems

- High cost of instruments, communication systems, and software presently used for MECposition monitoring

- Difficulty in tracking MECs if they break free because many MECs do not have satellite data feeds (radio and cell reception can be limited offshore and over long distances).

\section{Benefits of Closing the Gaps:}

- Low-cost MEC identification and monitoring system that uses off-the-shelf equipment and leverages the existing USCG AIS infrastructure

- Reduced risk to vessels

- Reduced risk of MEC loss and damage, resulting in reduced finance and insurance risks.

\section{Solutions:}

- Perform outreach/paper study to understand AIS requirements and eligibility and avenues to certify/standardize new technologies to meet AIS requirements and/or determine alternative solutions to achieve the same goal - this effort should also explore if systems other than GIS exist that fill the gap

- Develop a9 methodology of how AIS would be used in scenarios that range from a single MEC to a large MEC farm

- Work with the international standards bodies and USCG to implement procedures and policies for AIS implementations in marine energy applications.

\subsubsection{Methods and Guidelines for Near the Air-Water Interface Turbulence Measurement}

Turbulence has been demonstrated to impact the fatigue life of current (tidal, river, and openocean current) energy converter blades, hydrofoils, and other surfaces. Because turbulence is a measure of the energetic, rotational, and eddying state of water motion, as turbines move from protected passages to bodies of water with larger fetches, wave motion can affect turbulence measurement - it is hard to distinguish turbulence (rotational wave velocity) from wave-induced motion (irrational water velocity).

Methodologies and guidelines are needed to provide common procedures and measurement techniques on how to measure water motion and distinguish between turbulence and wave water motion at various depths based on mechanisms that may be creating the turbulence. Presently, the "structure function" method is the only known method of measuring turbulence within a wave field. Some acoustic Doppler current profilers (ADCPs) can be configured to operate in a high spatial resolution and low-noise mode with one beam oriented horizontally to use the spatial variations of the wave-induced velocity, which are smaller in the horizontal direction compared to the vertical direction. 
Gaps: Knowledge and techniques are somewhat limited on how to use existing instrumentation and process data to measure turbulence in a wave field.

\section{Impact of Gaps:}

- Incorrect or insufficient information is being collected to inform MEC design. This can result in bad design assumptions, an incorrect design basis, and flawed fatigue analyses, leading to either an over- or under-designed system — both of which increase costs.

- In atmospheric boundary layer flows (wind), turbulence has been extensively studied to determine turbulence characteristics for different terrain and flows. These data have been used to develop stochastic models in turbine design and site performance estimation. Without complete data sets for the range of metocean conditions, design capabilities for MECs will be limited.

\section{Benefits of Closing the Gaps:}

- Improved data sets that characterize turbulence in tidal flows will allow the development of turbulence models that can be used to design more robust and cost-competitive tidal turbine blades, structures, and power generation systems.

\section{Solutions:}

- Create literature review to determine if other methods exist to measure turbulence in a wave field

- Engage the oceanographic community to develop and field-verify better turbulence measurement techniques, instrumentation setup, and processing techniques that can identify and quantify turbulence in a wave field.

\subsection{Enhanced Measurement Capabilities}

\subsubsection{Affordable Mechanical and Power Take Off Load Sensors for Very Small- Scale Laboratory Testing}

Laboratory testing offers a first opportunity to characterize loads and validate performance predictions at small scales and early in the design cycle where issues can be addressed quickly at lower costs. Provided that correct physical model scaling is applied, small-scale loadmeasurement sensors could be mounted to small-scale energy converters at about 1:30 to 1:10 scale. These sensors would increase the knowledge gained from small-scale testing beyond what is currently gained, and that knowledge could be used to refine designs at smaller scales at much lower costs and shorter times than at later stages in the development timeline. However, mechanical load measurements are not often available at these smaller scales because appropriate commercial off the shelf (COTS) sensors may not exist or are too expensive. It is also difficult to produce sufficiently accurate and sensitive sensors that can measure loads on a small area and/or that have small enough mass so they will not affect the response of the device under test. Wires from these sensors can also increase a device weight and, hence, affect the device response.

Gaps: No COTS technology is known to be readily available and/or affordable to provide laboratory load measurements that meet the sensitivity and mass requirements for testing smallscale WECs. 


\section{Impact of Gaps:}

- Unreliable load data or inability to measure loads at small scale, thus limiting the utility of small-scale testing and requiring load testing to be performed at larger scales

- Increased project costs and development timelines because design iteration occurs at higher TRLs or required custom measurement solutions

- Affected device response by the additional mass and stiffness of load cells and cables from the sensors to the DAS.

\section{Benefits of Closing the Gaps:}

- Credible load measurements made at smaller model scales that provide a better understanding of loads, thus allowing more comprehensive design evaluation and design optimization at lower TRLs where design iteration is much less costly and much faster

- Improved load characterization, leading to higher reliability and better performance at later design stages and higher TRLs

- Utilization of additional data to calibrate and validate computer model

- Decreased impact on scale-model mass, leading to better response characteristics.

\section{Solutions:}

- Quantify the mechanical (structural and mooring) and PTO load requirements for small-scale testing and develop a specifications document

- Perform a survey of existing sensors and instruments to determine the range of measurement technology that is available and what measurement needs cannot be met with COTS sensors; update the MHK Instrumentation Database on what instruments/sensors are available and can meet the needs of load measurements for small-scale tank testing

- Work with manufacturers to make them aware of the unmet needs of load sensors for testing small-scale devices.

\subsubsection{Affordable Mechanical and PTO Load Sensors for Larger-Scale Field Testing}

Field tests provide critical data used to evaluate the performance and loads of MECs at scales where accurate or exact device response can be achieved. These data are used to validate assumptions, verify model results, estimate fatigue, refine design load cases and optimize future designs. Because the mechanical load-bearing structure accounts for a large share of CapEx, mechanical loads should be well characterized throughout the converter to provide sufficient information to optimize the design for cost and durability. Turnkey mechanical load measurement systems that can reliably function in the marine environment are often difficult to source and can be very expensive. Additionally, installation of load sensors can be difficult and require extensive work. For example, installing foil strain sensors requires special surface preparation, sensor mounting and coating, and waterproof wires to be run along the surface of marine converters. 
PTO system reliability is critical to maximizing availability and energy production. Component testing provides an opportunity to characterize normal and extreme loads, develop fatigue data, and identify issues such as vibration that may lead to premature failure. Load measurement on drivetrains can be difficult because of moving or rotating parts, high temperatures, exposure to lubricants, complex load paths, and tight access. Because of the high cost of some sensors and difficulties of specifying, sourcing, and installing mechanical and PTO load sensors, fewer load measurements are typically made than what are needed and failures in measurements can be unacceptably high. This leads to inadequate measurements and poor sensor performance and unreliability.

\section{Gaps:}

- No consensus turnkey solutions have been identified for measuring mechanical and PTO loads on large- and full-scale MECs.

- No guidelines or standards have been developed for measurement requirements and installation of mechanical and PTO load-measurement systems in the marine environment.

- Mechanical and PTO load sensors can be very expensive.

\section{Impact of Gaps:}

- Load measurement at a large scale can be very expensive and, because sensors can be costly to install, the number of measurements is often limited, thus reducing the number of important measurements available to guide future design optimization, leading to slower device design or designs that are suboptimal with reduced reliability.

- Installing sensors, such as strain gauges, for use in a marine environment can be difficult, and improper installation can lead to premature sensor failure.

\section{Benefits of Closing the Gaps:}

- Larger-range and higher-quality structural measurements at large/full scales that lead to better load (stress, strain, minimum, maximum, and fatigue) characterization and better information to inform future designs (optimization, reliability, survivability, availability, and $\cos t)$

- Accelerated design time and more robust designs. 


\section{Solutions:}

- Quantify the mechanical (structural and mooring) and PTO load requirements for large-scale testing for both test stand and field tests

- Develop a guidance document for installing and calibrating structural and PTO load sensors on WECs/CECs based on experience in the wind and ocean industry; verify the methods in laboratory tests and sea trials

- Perform a survey of a relevant set of existing sensors and manufacturers and update the MHK instrumentation database

- Create an inventory/library of load-measurement systems, such as fiber Bragg interrogators and rotary load cells, that can be loaned out to the MHK community to help facilitate load measurements.

\subsubsection{Wireless Time Sync of Underwater Instruments and Underwater Communication Between Measurement Array Elements}

Instruments used in field testing MHK technologies are often located in different locations (on the $\mathrm{WEC} / \mathrm{CEC}$, on the sea floor, in the water column, or floating on the sea surface). Because of the expense and difficulty of cabling measurement systems together, different measurement systems ranging from a single instrument to networked instruments often rely on different mechanisms to transmit data and synchronize clocks across standalone/unconnected systems. Measurement systems on the surface can use radio- (RF) and satellite (GPS)-based communication and synchronization methods. However, it is much more difficult to communicate data and synchronize the clocks of underwater measurement systems. Thus, they typically use their own clock to time stamp data and record those data internally. The clocks of the underwater systems are synchronized at deployment but operate independently thereafter and can drift apart. Data from the remote systems/instruments are typically collected at the end of the experiment and are not available for use during testing. Presently, there is no known solution to periodically synchronize of remote (not connected via a wired communication means) underwater instruments. The drift that results can mean it is very difficult, sometimes impossible, to align measurements in time. Data can be transmitted with acoustic modems, but they have very low baud rates and can use significant power; thus, acoustic modems require large sets of batteries and have limited life.

Gaps: Many underwater sensors, instruments, and DASs lack the ability to periodically synchronize clocks with a known standard and to communicate data between the instruments and a central data collection system. For underwater systems that use acoustic modems, they have higher power requirements, low data transfer rates, and limited deployment durations. 


\section{Impact of Gaps:}

- Clocks can drift, which creates difficulty with data re-alignment during post-processing. It is difficult to ensure data are comparable due to errors or uncertainties in the timing - this is particularly important for variables with a high rate of changes such as turbulence, loads, and power quality.

- Because underwater instruments without acoustic modems cannot communicate their data until they are recovered, there is a delay in processing those data, and any learning or knowledge cannot be gained during testing but instead must wait until the instrument is recovered. As a result, any opportunity to use learning to increase test impact and success is lost.

- Sensor/instrument failures cannot be detected during testing.

\section{Benefits of Closing the Gaps:}

- Temporally aligned data streams with continuous clock synchronization that eliminate temporal drift leading to higher confidence in the data

- Faster data post-processing with lower probability of errors, leading to higher confidence in data

- Near real-time data streams with the ability to perform on-the-fly sensor reconfiguration and immediate notification of sensor failure.

\section{Solutions:}

- Quantify the data transmission and clock synchronization requirements for offshore (e.g., communication distances between instruments used in MHK testing may be much less than those used for oceanographic measurement)

- Perform a survey of existing acoustic communication systems and their capabilities; update the MHK instrumentation database

- Work with the manufacturers of existing acoustic communication systems that do not meet the requirements of MHK testing or do not work with commonly used instruments so they can understand and meet the needs of MHK testing

- Explore low-bandwidth/low-power schemes, such as transmission of a regular acoustic sync pulse from a central node. The remaining elements of the acoustic network could receive only, which would lower power and processing requirements for all elements (except the central node)

- Explore underwater RF communications (WiFi) as an alternative; this has been recently demonstrated at short ranges $(<10 \mathrm{~m})$, but it is not yet commercially available.

\subsubsection{Standardized Time Sync of Cabled Instruments}

As MHK test facilities are established, cables are used to connect instruments and DASs to shore facilities to provide power to the offshore systems and to enable real-time monitoring and control. These cable connections provide a significant advantage because all measurements are networked together and can be coordinated to provide device developers and the test facilities near real-time information about the test, from the resource to the power being sent to the grid. 
Because instruments and DASs differ between projects and can even differ within projects, different time formats, clocks, time basis (e.g., local versus GMT), and clock synchronization methods may be used. This can make decoding the data difficult and lead to errors when aligning data sets. For data collected at high speed, such as for power quality or turbulence, it can be impossible to align data without tight synchronization between all measurement elements.

It would be useful for a standard time basis and for common clock synchronization methods to be established and adopted by test facilities to ensure tight synchronization of measurements using a consistent measure of time.

Gaps: Many underwater sensors, instruments, and DASs lack the ability to periodically synchronize clocks with a known standard and to communicate data between the instruments and a central data collection system. For underwater systems that use acoustic modems, they have higher power requirements, low data transfer rates, and limited deployment durations.

\section{Impact of Gaps:}

- Measurements may have different time formats and basis (UTC versus local time), making aligning data streams very difficult and resulting in a higher probability of processing errors when aligning different data sets.

- Clocks can drift, creating difficulty with data re-alignment during post-processing if they are not regularly synchronized with a common clock, such as the GPS clock.

- It is difficult to ensure data collected are comparable due to errors or uncertainties in the timing - this is particularly important for variables with a high rate of changes such as turbulence, loads, and power quality.

\section{Benefits of Closing the Gaps:}

- Temporally aligned data streams with continuous clock synchronization that eliminated temporal drift, leading to higher confidence in the data

- Faster data post-processing with lower probability of errors, leading to higher confidence in the data

- Fewer errors in design, leading to lower costs as over-/under-design is minimized.

\section{Solutions:}

- Develop guidelines for measurement synchronization of DASs and instruments in a test facility where cables are used to power and communicate with instruments and sensors used for WEC and resource measurement. 


\subsubsection{Mooring Line Load Cell with Robust Communications}

Mooring lines move relative to the WEC and are subject to high fatigue cycles and extreme loads, such as snap loading. Thus, cables that provide power and communications between the load cells and the WEC can fatigue. The relative motion between the WEC and the load cell can cause the load cell to impact/hit the WEC or can pinch the power and communication cable against the WEC or mooring hardware. During installation, it can also be difficult to protect the load cell and its communication and power cable. Load cells can be the weak link in a mooring system and may fail before the ultimate strength of other mooring components are reached.

Marine load cells exist, but they are cabled and have been proven unreliable for long-term deployment. Marinized load cells can also be expensive and use materials that are different than the mooring line, thus creating a galvanic couple - stainless steel to galvanized carbon steel.

\section{Gaps:}

- Mooring load cells exist, but they typically do not meet the reliability needs of MECs because they have been designed for applications with less body motion than what many MECs experience.

- Mooring line load cells used in offshore oil and gas are typically too large for MHK applications.

\section{Impact of Gaps:}

- Load cell failures are common (power and communication), resulting in limited data and often missing important events, thus leading to poor understanding of mooring loads and opportunities to improve design and performance.

- Cost of load cell replacement is high (if possible) because large vessels are typically required to separate the mooring line, replace the load cell, and reconnect the mooring line.

\section{Benefits of Closing the Gaps:}

- Reliable and high-quality mooring load data that can be used to help understand mooring design loads, thus leading to optimized mooring designs and optimized WEC systems

- Lower test cost

- Additional data to calibrate and validate computer models. 


\section{Solutions:}

- Determine mooring load cell requirements/specifications for WEC testing

- Perform a review of existing load cells to quantify existing capability (e.g., availability, cost, wired and acoustic communication, and specifications) and gaps for WEC testing; add relevant information to the instrumentation database (https://www.nrel.gov/water/mhkinstrumentation-sensor-database.html)

- Work with load cell manufacturers to determine the cost of adding the capabilities needed to obtain reliable mooring load measurements

- Develop short-range acoustic communication link and measurement sync between load cell and WEC if needed

- Perform third-party testing and certification of mooring load cells to verify their performance and reliability.

\subsubsection{Measurement in a High Electromagnetic Environment}

Marine energy converters are likely to generate localized electromagnetic fields that are much higher than ambient conditions. These fields can affect the performance of sensors and create noise in data transmission lines. This noise can overshadow analog sensor signals, thereby requiring significant post-processing efforts and reduced signal quality, if not rendering the signals useless. Digital signals/communication can also be affected such that data rates are reduced or communication is impossible. This can impact communication between instruments and a DAS as well as between the DAS and shore. Often, the level of noise is not known until the device is deployed and where fixes are very costly because they require diver intervention or recover and rework of the device under test.

\section{Gaps:}

- No recommended practices or guidance are known to exist to help the community properly protect/insulate sensors, instruments, and communication from the high EMI environment.

- Most underwater measurement technologies are designed for operation in the open ocean and do not have protection for high EMI environments.

\section{Impact of Gaps:}

- Potentially noisy data leading to lower quality and/or unusable measurements; results could range from longer test duration to test failure because the necessary data were not collected

- Unreliable and lower speed communications between DASs, instruments, and shore.

\section{Benefits of Closing the Gaps:}

- Higher-quality measurements that improve the impact of testing, lower testing costs, and improve testing success

- Reliable and higher bandwidth communications. 


\section{Solutions:}

- Develop a guidance document/recommended practice for sensor placement, signal protection, and signal conditioning based on national laboratories' experiences in testing wind turbines and on recommended practices and standards and place it on the MHK Instrumentation Database (https://www.nrel.gov/water/mhk-instrumentation-sensordatabase.html)

- Quantify the impact of EMI on different underwater sensors and publish results; if necessary, work with an instrumentation developer to modify sensors/instruments so they can operate in the high EMI environment when current sensors/instruments prove inadequate

- Establish a set of experts who can assist/review measurement systems to catch and fix any issues before deployment.

\subsubsection{Short-Term Wave Height Forecast at Device}

During WEC testing, wave measurements are typically performed in the far field where waves are not affected by the WEC or other obstructions. Because the wave profile evolves as the wave propagates, the time series at the wave measurement location and at the WEC are different. Also, because wave fields change spatially, if the wave direction of propagation is not in the direction from the measurement device to the WEC, or if the device is near shore where wave reflection and refraction occur, the measurement will not reflect the wave seen by the WEC. As such, conventional wave measurements made far from a WEC cannot easily be used to determine the future wave time series at the WEC. However, the ability to forecast the wave height time series at a WEC can have many benefits for device operation and survival.

In some cases, control system performance of WEC devices can be directly related to the ability to estimate wave time series at a device and estimate quantities such as the excitation forces acting on the device. Ideally, a WEC controller should be able to adapt, on a wave-by-wave basis, to maximize the energy extracted. However, this is not easy because ocean waves are irregular - nonstationary for short periods of time - and because they cannot be accurately forecast for more than a few seconds based on history. While not a trivial task, controller performance, hence energy capture, can be greatly increased with longer and more accurate wave height forecasts.

Wave height can change dramatically within a short time frame and, because they are composed of a range of wave components spread over a range of frequencies, they can sum constructively and events such as rogue waves can also occur. Without wave height forecasting, a device may need to go into a safe non-operating mode when conditions occur that may result in loads exceeding operating conditions; thus, limiting power production-AEP can be reduced. Wave height forecasts of several minutes or more could allow a device to reconfigure/move to a safe mode or take some form of preemptive action if a short-duration extreme event were forecast, thus allowing a device to operate in higher sea states and take pre-emptive action to avoid damage. 
Analysis of test data is also impacted because the wave time histories are not known (and do not actually exist) at the device. Without wave time series, time-series analysis cannot be used, and analysis is typically performed using spectral and statistical means. While characterization of the linear response and basic model validation can be performed using spectral and statistical methods under stationary conditions, the non-linear characteristics and analysis during nonstationary conditions and events (such as during storm events and rogue waves) is limited. The ability to estimate the wave field at the WEC from far-field measurements would allow timeseries analysis to be performed and, hence, allow more powerful non-linear data analysis tools to be used over a wider range of stationary and non-stationary conditions.

Presently, two systems are under development (one Office of Naval Research (ONR)-sponsored and one DOE-sponsored) that use wave radar to measure the incoming wave field and an analysis package that uses the wave field information to develop wave time-series forecasts at specified locations. The ONR system has been demonstrated to accurately predict the wave field for intervals up to 5 minutes at a location within the measurement area. ${ }^{2}$ These innovative technologies are being developed using shore and ship-mounted radar but are custom prototypes - no commercial system exists, and some elements of the hardware and processing tools are proprietary.

\section{Gaps:}

Radar-based wave forecasting concepts have been demonstrated but have not transitioned into commercial products, thus COTS systems are not known to exist. Buoy or other remote-sensing (e.g., Lidar) systems may also be viable but have not been demonstrated

\section{Impact of Gaps:}

- Because no COTS wave forecasting system exists that can forecast wave height time series at a WEC, WEC developers are unable to perform short-term wave-to-wave control.

- Survival strategies are limited to predominate conditions, resulting in reduced AEP, because without wave forecasts, WECs may be in a non-operational safe mode to avoid a lowprobability event when they could instead be production powers.

- Rogue waves can occur during safe operating conditions and without wave forecasting; a WEC cannot respond and go into a safe mode. This results in higher risk of damage to the device.

- Analysis of test data is limited to statistics and the frequency domain in stationary sea states.

\footnotetext{
${ }^{2}$ B. S. H. Connell, J. P. Rudzinsky, C. S. Brundick, W. M. Milewski, J. G. Kusters, and G. Farquharson. (2015). Development of an environmental and ship motion forecasting system. Proceedings of the ASME 2015 34th International Conference on Ocean, Offshore and Arctic Engineering.
} 


\section{Benefits of Closing the Gaps:}

- Increased energy production and AEP via better wave-to-wave control

- Reduced maintenance and increased availability when a WEC can respond to individual wave events to avoid damage yet otherwise operate

- Analysis of test data can extend to extreme conditions, unique events, and non-stationary conditions.

\section{Solutions:}

- Perform a literature review and develop estimates of the improvement for energy capture, AEP, and availability if wave forecasts were made at the device

- Work with the wave-forecasting technology developers to move the wave-forecasting technology from prototype to a commercial product with the needed capabilities for WEC control.

\subsubsection{Measuring Ocean Wave Elevations at the Device}

As discussed in the prior finding, time series of wave height time history measured in the far field are not the same time series seen by the WEC. While it is possible to measure the wave field directly at the WEC, the local wave field is subject to reflection, diffraction, and radiation due to wave-structure interaction at the WEC. As a result, any local wave height measurements will not be representative of the incoming wave field. It is possible to perform a local measurement from a stationary body of simple geometry and back out to the wave field; however, WECs tend to be complex shapes and directly interacts with the wave field making this task very difficult.

If wave time-series measurements can be made local to the device, wave predictions can be made based on history, but these forecasts degrade rapidly after 5-10 seconds - they cannot reach the same accuracy as the prior wave radar methods. However, the wave time series could make timeseries analysis of the wave-WEC interaction possible.

Gaps: Commercial and/or public tools for processing data and removal of reflection, refraction, and radiation are not known to exist.

\section{Impact of Gaps:}

- No COTS wave height time-series estimation methods or tools are known to exist for backing out of the incoming wave field from local surface height measurements at the device.

- Wave time-series short-term forecasting cannot be made using measurements from the WEC, and short-term (3-5 seconds) control would require additional remote sensors. This would increase project costs and lower energy capture

- Analysis of test data is limited to statistics and the frequency domain in stationary sea states. 


\section{Benefits of Closing the Gaps:}

- Increased energy capture and AEP via better wave-to-wave control-although this would be less than for longer-term 3-5-minute wave height time-series forecasting methods

- Analysis of test data can extend to extreme conditions, unique events, and non-stationary conditions.

\section{Solutions:}

- Develop methods to measure the local wave field at the WEC that compensates for device motion

- Develop the theory and techniques for removal of the reflected, refracted, and radiated waves from local wave field measurements at a WEC

- Perform field validation of the methods and characterize the accuracy over excepted sea states and device motion.

\subsubsection{Increased Data from Existing Instruments}

Many instruments used in MHK-related field and laboratory measurements were developed in support of other industries and in different areas of scientific studies. In many cases, these instruments were developed to perform a specific measurement that coincides with MHK data needs, and they have thus been adopted by the MHK community. The instruments use onboard processing to convert raw signals to useful engineering data; however, many of the raw signals may also contain information that can be processed in different ways or have their sampling methodology changed to provide additional information that can meet other MHK-measurement needs. A classic example is the acoustic Doppler profiler (ADP). The ADP was originally used to provide a water-speed profile via acoustic Doppler measurements. By adapting the processing, ADPs were enhanced and are now used to calculate directional wave spectra using the measured orbital water velocities caused by waves. There are many other examples where raw data contain additional information that can be used to provide more data, including inferring bottom substrate using the echo intensity from depth sounders and multi-beam sonar, determining machine health using structural load accelerometers or ambient acoustic transducers, sea state using device motion sensors, and short-term wave forecasting using conventional ship radar.

Processing within existing instruments can also be extended to add additional information about the measurements such as an uncertainty estimate. While this can be done in post-processing with information from the manufacturer, a built-in capability would provide information useful for bankable resource assessments and device certification.

Because the MHK industry have leveraged instruments from other fields (e.g., oceanography, marine biology, and offshore oil and gas), these instruments have not been designed to meet the often-unique needs of the MHK industry. Thus, there is an opportunity to work with instrument developers to customize software to provide additional data and, hence, increase the information gained during testing. 
Gaps: Instruments have not been optimized to meet the measurement needs of the MHK industry, and thus, they may not be processing their measurements to provide the full range of information to users that are contained in their raw signals.

\section{Impact of Gaps:}

- Because instruments often only provide processed data and not the raw signals or access to the internal code, there is not opportunity for users to customize the processing of the raw data to provide additional information. Thus, additional sensors may need to be purchased or that additional information may not be calculated.

- If an instrument provides raw signals, it is often very difficult for users to interpret the signals and develop the processing code. This can add cost and time to the project or that additional information may not be calculated.

\section{Benefits of Closing the Gaps:}

- For testing, when an instrument can provide the processed signal that includes all possible relevant MHK testing information contained in the raw signal, this will reduce the costs associated with monitoring both device and environmental characteristics, and in doing so, it can provide a wider range of measurements to help guide future design and permitting or information that can be used in health monitoring that reduces operations and maintenance (O\&M) costs.

- For site characterization, the additional measurements can provide accelerated learning, and the increased information can be used to broaden the site characterization and help permitting.

- There is an opportunity to reduce the number of instruments used in testing and resource characterization if an instrument's raw signals can be processed to produce the information from multiple sensors. Again, using the ADP example, the instrument can measure both the current velocity profile and wave field, thus eliminating the need for separate wave and current sensors.

\section{Solutions:}

- Review the different instruments and their measurement methods used in MHK site characterization, resource assessment, and testing; determine if there is a possibility to extract additional information from the measurements that either provide data for unmet measurement needs or can replace separate instruments - this step should include a literature review to determine the state-of-the-art and identify upcoming technologies

- Work with the instrument manufacturers to determine the possibility of performing additional processing to yield the new data capabilities

- Work with universities or instrumentation manufacturers to develop new processing methods if needed. 


\subsubsection{Use Existing Sensors to Monitor Machine Health During Testing}

Field testing of MECs employ a wide range of sensors, including strain gauges and accelerometers to measure the structural and PTO response, acoustic hydrophones to measure radiated noise, and very high rate power transducers to characterize power quality parameters. Often these sensors are the same sensors used in health monitoring, and/or they measure parameters that can be used in health monitoring. Often, health monitoring is not a test goal, yet because many of the same parameters are measured, testing can be used not only to tune/train health-monitoring systems but also to perform health monitoring. Conversely, if a healthmonitoring system is used, it may use separate sensors. Combined test measurements and health monitoring can have significant benefits during testing because it can detect issues that arise that can be more quickly solved and lower costs before the issue manifests in a failure.

During testing, a wider range of measurements is often made than what would occur for a commercial operation. These include acoustic noise measurements, which are needed as part of permitting and likely certification. These measurements also provide a capability to characterize device health.

Gaps: Health monitoring is not performed during testing of MECs or separate health-monitoring systems are used that may duplicate instruments.

\section{Impact of Gaps:}

- Malfunctions may not be identified early enough to prevent failure. This can increase testing cost and duration, as well as have a negative impact on the credibility of the MHK industry.

- When separate test and health-monitoring measurement systems are used, measurement complexity and test costs increase.

- Test-specific measurements, such as acoustic noise, can be used to help characterize device health and tune health-monitoring systems, while efforts in this area exist for many test campaigns, analysis is not done in real time or does not directly integrate information that can help detect issues. Thus, any changes in system health may not be noticed during testing.

\section{Benefits of Closing the Gaps:}

- A greater understanding of the symptoms of a fault can be developed and used to advance early detection in support of condition-based maintenance.

- Early identification of faults, so maintenance rather than replacement is possible, can result in lower test cost, shorter test duration, and higher test success.

- Measurements can be used for multiple purposes, thereby reducing measurement redundancy and testing costs.

- Health-monitoring systems can be developed during lower TRL testing instead of at higher TRLs. 


\section{Solutions:}

- Quantify and document the measurement requirements of health monitoring for a range of MECs; determine what measurements are common to both

- Work with test centers to develop guidance documents and encourage real-time health monitoring of test articles

- Develop a DAS able to support the data acquisition needs and the processing needs for both testing and health monitoring - this may include adding health-monitoring capabilities to MOIS and other commonly used DASs.

\subsubsection{Micro DAS}

Testing at small scales at lower TRLs offers technology developers a first chance to evaluate the response and performance of their concept prior to proceeding to more expensive and largerscale tests at higher TRLs. For a scale model to accurately represent the dynamics, loads, and performance of the proposed full-scale system, the mass, inertias, and stiffness must all be accurately scaled. Unfortunately, for smaller-scale WECs, from 1:10 and smaller, adding an onboard DAS is often not an option because the mass and location of the DAS changes the mass and inertial properties of the body. When DASs are not mounted on the WEC, wires are run from the sensors to the remote DAS, and the mass and stiffness of the wire bundles alter the WEC response. Because of these impacts on mass, inertia, and stiffness, physical models are limited to larger scales or, as small scales, the type of tests run are limited, and the quantity and quality of measurements are reduced. As well, the data from small-scale testing can be questionable because of the changes in inertial properties and because the signal conditioning may be a distance away from the sensors, thus limiting the confidence in smaller-scale test results. If the mass and size of the DAS can be significantly reduced by leveraging advances in dataacquisition technology, a wider breadth of testing could be conducted more accurately at smaller scales at much lower costs and time than at later stages in the development timeline.

Gaps: No turnkey COTS micro-DAS hardware and software are known to be available and affordable.

\section{Impact of Gaps:}

- Developing custom DAS for small-scale device testing is a specialized field. Time and effort spent by technology developers on developing small-size DAS equipment would be better spent performing tests.

- Without a small DAS, the device response is affected by the additional mass and stiffness of the cables from the device under test to the DAS. Early-stage erroneous testing can lead to false conclusions and misguided future design. Once the erroneous results are identified at larger scales, costly redesigns may be required, causing delays in progress toward commercialization.

- Some signal degradation can occur when long wires connect the sensor to the DAS. 


\section{Benefits of Closing the Gaps:}

- Improved device response at smaller scales because of a reduced number of cables and a DASs of negligible mass, leading to higher-quality information to feed future designs (optimization, reliability, survivability, availability, and cost); this would allow more comprehensive design evaluation and design optimization at smaller scales and lower TRLs where design iteration is much less costly and much faster.

- Improved signal quality for measurement channels.

- Lower testing costs because device developers could utilize a common system instead of developing custom solutions.

\section{Solutions:}

- Quantify the measurement needs and dimensional/mass requirements for a small-scale DAS

- Perform a survey of existing small form-factor DASs and quantify their capabilities against the requirements

- Create a micro-DAS design and software framework, including identification of the DAS hardware

- Build the DAS and software, test, and make the system available to the MHK community`.

\subsubsection{High-Speed Underwater Communication for Tank Testing}

Many WEC concepts are fully submerged, and this limits the methods of communication to an external data logger and other test equipment, such as real-time displays. Presently, cables are the most common method used to communicate with onboard sensors and DASs; unfortunately, these cables change the mass and stiffness of the WEC, leading to altered inertial and stiffness characteristics. RF communication is possible, but this would require an antenna to protrude from the water, thereby affecting the device response. Acoustic communications may offer a potential solution of transferring data and control commands between a WEC model and the external testing hardware. While underwater acoustic modems exist, they are designed for openocean applications - not laboratory tanks. These systems have much lower communication speeds than needed for laboratory testing because they are designed for a noisy ocean environment with stratified water, complex terrain, and long-distance transmission. In a test tank, the communication distances would be much smaller and the environment easier to transmit data without noise and multi-path; this could allow for much higher bandwidths and lower power requirements when compared to open-ocean acoustic communications. Another option is a highspeed optical communication link using a laser to transmit data. Regardless of the form of the non-cabled communication, any solution would likely require an onboard DAS to acquire and digitize data, encode, and then transmit those data.

Gaps: COTS high-speed underwater wireless communication technology designed for short distances in the simplified laboratory environment is not known to exist. 


\section{Impact of Gaps:}

- Innovative concepts such as underwater WECs cannot be tested at smaller scales without the response and performance being impacted by the communication wires. This limits the breadth of testing that can be conducted at small scales and lower TRLs, thus increasing project costs and development timelines because design iteration will occur at higher TRLs.

- The device response can be affected by the additional stiffness of the cables from the WEC to the external test equipment, leading to inaccurate data. Early-stage erroneous testing can lead to false conclusions and misguided future design. Once the erroneous results are identified at larger scales, costly redesigns may be required causing delays in progress toward commercialization.

- Some signal degradation can occur when long wires connect the sensor to an externally mounted DAS.

\section{Benefits of Closing the Gaps:}

- Improved device response at smaller scales because of a reduced number of cables

- Improved signal quality for measurement channels if a DAS is used onboard the WEC

- Lower testing costs

- Higher-quality information to feed future designs (optimization, reliability, survivability, availability, and cost)

- Comprehensive design evaluation and design optimization at smaller scales where design iteration is much less costly and much faster.

\section{Solutions:}

- Quantify the communication (data and control) needs for testing WECs at scales up to 1:10

- Perform a survey of existing acoustic and optical communication systems to identify candidate technologies

- Work with universities and manufacturers to develop a solution that meets the needs for WEC laboratory testing.

\subsubsection{High-Accuracy 6DOF Measurements (On Device)}

Motion measurement of scale physical models of WECs in six degrees of freedom (6DOF) (surge, sway, heave, roll, pitch, and yaw) is critical to fully characterizing the response and for providing sufficient data to validate numerical models. Accurately measuring the motion of WECs can be difficult because they may consist of multiple bodies, be submerged, be flexible, be long in comparison to tracking capabilities, experience periodic overtopping/submergence/green water events, or be a combination of some or all of these. Conventional solutions to motion measurement include optical tracking, inertial sensors (motion reference units, MRUs/inertial measurement units, IMUs), and displacement sensors. Each of the sensors have limitations. Optical sensors work well for one or more bodies above the water surface but may not be able to cover the full test area. Trackers can be obscured (by water, test apparatus, or other bodies), and they may not work underwater. Optical tracking may also not have the required accuracy and update rates for testing smaller models; additionally, the tracking 
markers might affect the inertial and performance properties of the WEC. Inertial sensors offer higher measurement rates, but they suffer from bias, and drift, both of which can cause the integrated signals to rapidly degrade. Performance of inertial sensors also tend to degrade with smaller units being less accurate. Small 6DOF IMUs have not met accuracy requirements in tank testing, and thus, using higher-accuracy (larger and heavier) sensors may change the mass and inertial properties of a test article. Displacement sensors such as optical encoders and linear/rotary potentiometers can measure the relative motion between bodies but are typically not used to measure the absolute position and motion of a WEC.

Gaps: There is no known affordable turnkey COTS solution for high-accuracy measurement of 6DOF motion for the range of WEC archetypes and scales of physical models. Thus, test facilities are limited in their ability to make 6DOF measurements reliably, repeatedly, and accurately.

\section{Impact of Gaps:}

- Inaccurate motion data, especially at small scales and for underwater devices, can lead to false conclusions and misguided future design. Once the erroneous results are identified at larger scales, costly redesigns may be required, causing delays in progress toward commercialization.

- Inertial sensors and optical trackers can affect the device response by adding mass and changing the device inertial properties.

\section{Benefits of Closing the Gaps:}

- Reliable, high-rate, and stable motion data for above- and below-water WEC bodies can be used to help understand motions and device response for smaller models, thus leading to optimized WEC systems at lower TRLs.

- Accurate motion measurements provide additional data to calibrate and validate computer models, leading to higher confidence in these models. This higher-quality information can then be used to feed future designs (optimization, reliability, survivability, availability, and cost).

- Better motion data allow for comprehensive design evaluation and design optimization at smaller scales where design iteration is much less costly and much faster. 


\section{Solutions:}

- Quantify the motion measurement needs for testing WECs at scales up to 1:10

- Perform a survey of existing measurement systems to identify candidate technologies and quantify their capabilities

- Work to develop solutions that meet the motion measurement needs, and these solutions could be:

- A data fusion algorithm that integrates IMU and optical tracking measurements to obtain high-rate accurate motion measurements with elimination of long-term drift - this can leverage algorithms developed in sensor fusion

- An optical tracking system capable of simultaneous underwater/above-water motion measurement

- A new or more advanced IMU/MRU that meets the 6DOF measurement needs of MHK testing.

\subsection{New Measurement and Testing Capabilities}

\subsubsection{Black Box to Record Data for a WEC Device (Health Indicators)}

The MHK field is establishing credibility through adoption of international standards, establishing recognized test centers and successful demonstrations of new technologies. Part of this effort needs to include consistent, traceable, and verifiable data sets on device performance, reliability, and safety. This type of information is critical at the early stages of the industry to help improve designs, inform investors, achieve public acceptance, and provide information to regulatory agencies. A self-contained "black-box" DAS with independent third-party sensors installed on a device could provide data toward establishing credible baselines for MECs. A robust black-box system that can survive submergence and other catastrophic failures would also be useful for forensic analysis in the event of a catastrophic failure.

An acceptable black-box data recorder would need to be:

- $\quad$ built by a third party with knowledge and experience making measurements relevant to MHK testing,

- use high-quality components and sensors with traceable calibrations,

- be robust with verified performance and reliability, and

- be built to survive all expected failures.

Government sponsors, investors, and risk managers as well as project developers and utilities will benefit from an industry-accepted third-party DAS, display interface, and analyses to enable data-driven decisions. A suite of standardized DAS packages from a national laboratory with defined data sets and available technical services will reduce risk and accelerate the path to commercialization. 
Gaps: A COTS self-contained black-box system that can measure performance, loads, motions, and other parameters relevant to MECs is not known to exist. Without a third-party black-box system that uses independent sensors (not installed by the device developer), test data are often only provided using the developer's DAS and sensors.

\section{Impact of Gaps:}

- Limited data sets of unknown quality can be perceived as biased if made by the device developer. Insufficient data sets can limit understanding of performance and response, leading to longer development times and higher costs. Risk managers, investors, insurers, and regulators may be underinformed and may not fully trust the data, leading to project delays and decreased funding.

- Without a hardened measurement system that can reliably store data after a device failure, forensic analysis may be limited in the event of the loss of a device, and the causes of failure may not be determined.

- MEC developers need to develop their own instrumentation packages, leading to higher costs, longer development times, and likely fewer measurements because of tradeoffs between costs.

\section{Benefits of Closing the Gaps:}

- Credible third-party data sets for performance, reliability, and safety could lead to faster acceptance of technology and higher investor confidence.

- Data sets that are consistent across MHK archetypes could allow creation of performance baselines, direct comparison of performance and reliability, and creation of common data sets for model validation.

- With a robust system that can preserve data even after a catastrophic event, forensic analysis could possibly lead to quicker understanding of design failures and advance robust designs.

- Consistent third-party data sets could assist the permitting process and public acceptance via independent credible data sets that can be archived and shared to assist in future environmental evaluations

- A third-party black-box DAS tailored for testing MECs could provide test facilities and technology developers with a turnkey solution to field measurement, thereby reducing development timelines and costs. 


\section{Solutions:}

- Quantify the measurement needs for field testing MHK technologies and the requirements to survive catastrophic device failures

- Perform a survey of existing measurement systems to identify candidate technologies and quantify their capabilities

- Adapt existing DASs for this application; develop strong housing with robust connectors that can survive catastrophic events

- Develop a suite of standardized DAS packages, with defined data sets and available technical services similar to those of the NREL MOIS program.

\subsubsection{Underwater Pressure Grid for Tank Testing}

WEC technologies are subject to wave loads on their absorbing elements and across the device structure. The wave-induced pressures can vary across a surface and cause asymmetric loading and fatigue. To fully understand and characterize these loads, the pressure distribution across a WEC's surface must be measured to a spatial resolution that captures the load variation. These measurements can be used to help optimize the structure design — often the highest component of CapEx. Pressure taps are a common method to measure such pressure distribution, but they require tubes connecting the pressure port to a pressure gauge. This solution is impractical because the many pressure tubes needed will impact the device response.

A sensor is needed that can measure the pressure force with a high resolution, sensitivity, and dynamic response. It should be able to conform to the complex shapes of WECs. It should also be sufficiently light so it does not affect the response of the WEC.

Gaps: No COTS sensor is known to exist that can measure the pressure distribution of waves impacting WECs. As such, test facilities are unable to directly measure the pressure distribution on the surfaces of a WEC.

\section{Impact of Gaps:}

- Unable to measure the pressure force distribution on physical prototypes, leading to poor understanding of wave-induced forces, and limited capability to characterize wave forces and fatigue on physical prototypes; this will likely lead to higher design margins, resulting in increased structural costs.

- Increased project costs and development timelines because design iteration occurs at higher TRLs where pressure and structural loads can be measured at larger device scales

- Unable to validate the wave loading on numerical models. 


\section{Benefits of Closing the Gaps:}

- Credible data that can provide a better understanding of wave-induced loads at smaller scales, thus allowing more comprehensive design evaluation and design optimization at smaller scales where design iteration is much less costly and much faster

- Improved load characterization, leading to higher reliability and better performance at later design stages and higher TRLs

- Accurately characterized wave loads on scale models to calibrate and validate computer models, as well as develop a better understanding of the accuracy and limitations of WEC numerical models, and to identify areas for improvement

- Increased credibility and acceptance of computer models because of higher-fidelity calibration and validation.

\section{Solutions:}

- Develop the specifications (e.g., grid resolution, measurement range, frequency response, and mass limitations) for measuring the pressure distribution on different WEC model scales

- Review technologies for measurement of pressure distribution over a small area

- Design, build, and test an underwater pressure grid.

\subsubsection{Generic Control DAS, Sensor, Signal Conditioning, and Data QA Package}

Control systems used in laboratory and field testing use a wide range of sensors, including 6DOF device motion, wave time series, mooring loads, and grid simulators - many of these types of measurements are common to most controllers. Many controllers require good signalconditioning systems to remove spurious data, sample jitter, and drift, as well as to appropriately condition signals; otherwise, a controller might go unstable or perform poorly even though the control theory is sound. Data QA is needed to identify and flag questionable measurements. Finally, many advanced controllers require foreknowledge of the wave field at the location of the WEC so they can perform wave-to-wave control. During both field and laboratory testing, device developers are required to develop their own sensor and DAS package. Unfortunately, developing a DAS with the range of measurements and necessary real-time signal conditioning can be very expensive and require significant development time. Often, full sensor and system integration must happen at the test site or test tank. This step can require significant time and often leads to test delays as issues with sensors, communication, and integration of the test facility equipment with the WEC are troubleshooted - especially if the system consists of multiple DASs without common signal synchronization. If each test facility (laboratory and field) had a robust DAS with onboard signal conditioning that provided the most common signals used in WEC control, testing risk, cost, and duration could be significantly reduced. A focused DAS could develop a system that has other benefits such as a distributed DAS with one master system and many slave devices, which can help significantly reduce hardware cost and allow for shorter sensor leads that reduce signal degradation between the sensor and digitizing element. 
Gaps: No turnkey DAS with onboard processing is known to exist at laboratory and field testing facilities to provide common signals used in control of WECs.

\section{Impact of Gaps:}

- Developing a DAS to support active WEC control is time intensive and needs many iterations to get working correctly when onsite and setting up for testing. This can add significant setup time, cost, and risk to the project.

- DAS development efforts may be duplicated between projects, even when the same test facility is used. When unique sensor networks are used (but they may provide the same data measured in other tests), test facilities cannot leverage experience gained in prior tests.

- Erroneous data can lead to poor or unrepresentative/suboptimal controller performance, thus leading to false conclusions and misguided future designs. For smaller-scale tests, once the erroneous results are identified at larger scales, costly redesigns may be required, causing delays in progress toward commercialization.

- Without data QA, there could be a lag between the first occurrence of a data issue and identification of this issue. This could result in unrepresentative controller behavior and repeating tests once issues are identified and fixed leading to longer development times and increased testing costs.

\section{Benefits of Closing the Gaps:}

- If the measurements are provided by a robust DAS developed and verified during prior tests, during pre-test development, the WEC developer can simply use data specifications and sample data from the DAS to develop their controller. Once at the test site, they could set up in a plug-and-play fashion and hopefully skip the sensor integration and troubleshooting step. This can result in faster test setup and test execution because data streams are already appropriately conditioned, thereby decreasing test duration and costs.

- Reliable data streams with QA provided to the controller will increase confidence in the control performance and could reduce test duration because measurements are more reliable so any issue could be identified when they occur and then fixed.

- Higher-fidelity control systems allow better evaluation of control systems at smaller scales. 


\section{Solutions:}

- Develop a guidance document for WEC control system measurement and signal-conditioning specifications based on prior test experience (e.g., Sandia's control project) and expected future control systems for both laboratory and field testing

- Develop a design for a DAS with associated signal conditioning for laboratory WEC control - this should also leverage experience such as from Sandia's control project and NREL's field measurement

- Encourage test facilities to develop a core set of measurements that WEC developers can use for control

- Encourage/demonstrate system and DAS prototyping and testing methods to better ensure successful performance during deployments.

\subsubsection{Help Implement Control Systems}

Control systems can be difficult to implement, yet they are critical elements that impact the performance of wave energy devices. Because controllers can be complex and require accurate knowledge of the device state and the operating environment, there are many areas where controls can go wrong. For example, as stated in the prior finding, the combination of a poor design in one or more of the PTOs, controllers, and DASs can cause a controller to go unstable, even though the controller is theoretically functional and well designed. This was highlighted in the 1:20 scale tests of the wave energy prize where the controls were often the most difficult part of the test to get working, and many contestants had to use de-rated controls for their concepts to function.

Having good design may not be more expensive than bad design, and using the correct tools and techniques to design and analyze a closed-loop system prior to testing is critical and can help mitigate issues and help maximize controller effectiveness - the DAS should not be treated as a standalone part of the control development.

Ideally, test setups should be designed to minimize the systematic error that may occur because of measurement, signal conditioning, and the test environment so that WEC developers can focus on testing and tuning their control theory instead of chasing other errors and issues that affect controller operation. The DOE national laboratories have extensive experience integrating DASs with controllers in MHK and other fields such as wind turbine control (although wind control is substantially different than WEC control). DOE can help the success of testing MHK controllers if they are able to provide support for implementing control systems on physical models (integration of sensors, actuators, hardware, and software). This could include expert guidance to implement control systems on scaled devices and provide discounted software licensing for developers (MATLAB/Simulink).

Gaps: Control systems need to work properly during testing to get full use of tank tests and verify the performance of controllers. Design and integration of the PTO, controller, and DAS must be carried out concurrently and presently; control systems are one of the most common pain points during testing, and obtaining expert guidance with hands-on experience is difficult. 


\section{Impact of Gaps:}

- Integrating a DAS and controller is difficult, time intensive, and needs many iterations (often done by trial and error) to get working correctly for developers with limited expertise in this area. This can add significant setup time, cost, and risk to the project.

- Knowledge and experience from past DOE projects is not sufficiently leveraged to support existing and future work leading to repeated mistakes and longer development timelines, higher testing costs, and poor performance of a controller that may have otherwise been acceptable.

\section{Benefits of Closing the Gaps:}

- With support from national laboratory experts with experience integrating control and DASs, faster test setup and test execution can be achieved because knowledge can be leveraged from past projects to help future projects succeed, thereby decreasing test duration and costs.

- Testing higher-fidelity control systems can be performed using a more robust DAS, thereby allowing better evaluation of control systems at smaller scales.

\section{Solutions:}

- Develop a guidance document and lessons learned for testing WEC control system and integration of WEC controllers with measurement and signal-conditioning specifications based on prior test experience and place it on the MHK Instrumentation Database (https://www.nrel.gov/water/mhk-instrumentation-sensor-database.html)

- Involve DOE national laboratory subject matter experts in the design at an early stage to provide review and feedback to support the development and integration of control systems with measurement streams for tank testing; encourage development teams to include expertise in control, data acquisition, and signal processing

- Hold workshops and training sessions for design and integration of PTO, control, and DASs.

\subsubsection{Increase Availability and Accessibility of Instrumentation}

Oceanographic, environmental, mechanical load, motion, and performance instrumentation are critical aspects of all field tests because they provide the data needed for resource assessment, device characterization, and feedback on device performance that can be used to guide future design optimization. Unfortunately, marine grade instrument costs can be a significant portion of offshore resource assessment and testing budgets. Some ocean instruments can cost well over $\$ 100,000$ when support equipment, such as acoustic releases, deck boxes, anchors, and buoys, are included. As such, it can be very difficult to purchase the necessary sensors on limited budgets, and cost tradeoffs may be made that reduce the number of instruments and quality of instruments in exchange for maintaining other portions of the project. Often, once a test is over, instruments purchased to support the test may see limited use, and new projects may purchase the same instrument. The net result can be increased program-wide costs yet limited data sets. 
The DOE national laboratories have a significant inventory of instruments and support equipment that have and are being used for resource assessment and device testing. A few of these instruments include oceanographic instruments, meteorological instruments, the MOIS DAS, motion reference units, fiber optic interrogators, GPSs, acoustic releases, acoustic deck boxes, and power sensors. Because DOE already owns a large inventory of instruments, it would be beneficial to establish a library of DOE-owned instruments that can be used to facilitate nocost loaning to DOE and other DOE-funded MHK projects to support a wide range of measurements and data collection. As part of maintaining the library, the national laboratories would keep the instruments calibrated and maintained to ensure that they are ready for use. The DOE national laboratory staff could also provide knowledge and guidance in the use of the instruments to help maximize the success of measurements.

There are a large number of institutions (universities, research laboratories, and companies) that may be willing to loan or rent measurement equipment. Thus, the instrumentation database should also include a list of owners (who are willing to loan or rent) for each type of instrument in the database.

Gaps: The high cost of marine instrumentation makes it difficult for the MHK developers and researchers to afford and, hence, acquire the necessary instruments that meet the measurement needs of a project.

\section{Impact of Gaps:}

- Missed opportunity to leverage existing instruments via loaning/leasing/rental instead of purchasing such instruments, resulting in increased testing and resource assessment costs and perhaps reduced measurements.

- Fewer measurements made during testing, leading to reduced and/or incomplete data sets. This can have a significant impact when devices cannot be fully characterized or causal relationships cannot be fully understood. This leads to less knowledge supporting future design iterations, longer technology development, and higher costs.

- With the high cost of quality instruments, developers and test centers may choose to acquire lower-quality instruments, which could result in lower-quality data (e.g., poor resolution, stability, and accuracy) and/or data gaps because of higher failure rates. 


\section{Benefits of Closing the Gaps:}

- If instruments can be borrowed from the instrument library or leased from a third party, testing costs could be significantly reduced and the breadth of measurements increased because of ready access to the needed instruments. By channeling future DOE-sponsored instrument purchases into the library, a significant national asset can be developed that supports present and future MHK development.

- National laboratory staff would maintain and calibrate instruments to ensure they are ready for use and able to make accurate measurements.

- Involvement of DOE national laboratory staff in helping developers select and set up instruments will ensure that the correct instruments are used and operated. This also ensures that lessons learned from prior deployments are used in future testing.

- The MHK instrumentation database with modifications that identify instrument owners who can loan/rent equipment could reduce cost but also increase collaboration.

\section{Solutions:}

- Establish an instrument library at a national laboratory to loan (no cost to DOE-funded projects and facilities and perhaps a small fee for non-DOE-funded work) and maintain instruments in support of future DOE-funded projects.

- Have DOE maintain ownership and control of high-capital instruments purchased using DOE funds; if an instrument is purchased for a project, once that project is complete, the instrument should be returned to DOE and placed in the library to support future projects

- Add additional capabilities to the MHK Instrumentation Database that will allow users to add instruments that they are willing to loan/rent; DOE national laboratories should update their instrument inventory within the database.

\subsubsection{Design Grade Metocean Studies and Tools for the Determination of Site- Specific Metocean Parameters for the West Coast, Hawaii, and Alaska}

In the early stages of MHK design, in site selection, and during MEC operation, knowing the probability of occurrence of sea states throughout the year is critical. Knowing the occurrence of specific sea states allows developers to identify the weather windows for which they can operate offshore and perform deployment and maintenance activities. Data such as exceedance tables, persistence estimates, return periods, and wind and wave roses can help developers determine if a site is suitable for their technology and identify time frames when they can perform offshore work. These data can also be used to inform a design basis. Directional wave spectra are critical information for device and site developers to make credible predictions of operating loads and energy production, estimate device responses, and optimize device design and control to maximize energy production and reduce the levelized cost of energy (LCOE). 
The offshore oil and gas industry and metocean companies have developed detailed site- and area-specific data sets for exceedance tables, persistence estimates, and return periods for the Gulf of Mexico. The U.S. Army Corp of Engineers has also developed an online tool (http://wis.usace.army.mil/) that presents such data for many locations along the U.S. West Coast, Hawaii, and Alaska. While the U.S. Army Corp provide useful data, they are insufficient for a complete design basis and may not be at the specific sites needed by a developer.

Many tools exist that ingest metocean time series and spectral data and run statistical analyses to calculate site-specific metocean parameters, but they tend to be in-house codes and not public open-source codes. Thus, vetted public tools/source code would help developers because data from wave buoys and wave hindcast models exist that have sufficient information that would allow them to produce their own site-specific, detailed data sets.

Yearly statistics are suitable to establish a design basis, estimate AEP, and perform technology performance level (TPL) assessments at low TRLs. However, monthly metocean parameters should be available for the locations likely to see early development, including the U.S. West Coast, Hawaii, and Alaska, to support planning of O\&M strategies and estimate project costs.

Gaps: Publicly available tools/code that use accepted statistical analysis methods and that have been vetted by technical experts to calculate sea-state exceedance tables, persistence estimates, return periods, wind and wave roses, and representative directional wave spectra data sets are not known to be in use within the community. Additionally, public data for monthly sea-state exceedance tables, persistence estimates, return periods, and representative directional wave spectra for locations that are likely to see early development are not available.

\section{Impact of Gaps:}

- Estimated design loads, annual average energy production, and O\&M requirements (such as equipment and time frames) need to be known at early stages of WEC design to ensure that the technology will be appropriately designed and optimized for the site and to obtain accurate estimates of LCOE. Without site-specific metocean parameters that reflect the monthly variability of the sea states, there is increased uncertainty in WEC design, likely leading to higher failure, health and safety issues, longer time to commercialization, and higher development costs.

- Site selection is difficult and without site-specific metocean parameters that reflect the monthly variability of the sea states, site developers may not select the best WEC technology or might choose a suboptimal site to develop.

- Accurate estimates of annual wave energy production are also important when forming a bid on a site lease, when negotiating power purchase agreements, and when designing a site. This task becomes much more difficult and uncertain without site-specific metocean parameters that reflect both yearly and monthly variability of the sea states.

- Without publicly available tools that ingest metocean data and calculate site-specific metocean conditions that have been vetted by subject matter experts, the MHK industry will develop their own tools to process buoy and hindcast data, leading to duplicate tool development efforts, higher project costs, and possible errors in the analysis. 


\section{Benefits of Closing the Gaps:}

- Having public tools to calculate site parameters such as exceedance tables, persistence estimates, return periods, and wind and wave roses from existing metocean data would help developers, researchers, and the community to perform independent design grade metocean studies.

- Having public metocean data and pre-calculated site parameters (exceedance tables, persistence estimates, return periods, and wind and wave roses) - perhaps in the form of a GIS-shape file at a similar spacing to the MHK wave atlas (https://maps.nrel.gov/mhk-atlas/) with temporal resolutions of one year to monthly for the U.S. West Coast, Hawaii, and Alaska - will help reduce costs and accelerate MHK technology and early site development by:

- Providing information that can be used to formulate realistic design bases, thus yielding WECs that are designed for the conditions to be experienced in the United States

- Improving estimates of average annual energy production, leading to better estimates of LCOE, better conditions for power purchase agreements, and reduced uncertainty for site bidding

- Helping site developers select that best technology for a given site or select the best site for a given technology

- Providing information that can be used to determine O\&M scenarios and the requirements for supporting equipment.

\section{Solutions:}

- Locate and catalog public tools for processing data from wave buoys and hindcast models to calculate site-specific metocean parameters; then identify gaps in the public tools

- Develop tools that fill the gaps in the public tools for calculation of site-specific metocean parameters and place them in the online code repository

- Perform design grade metocean studies that process buoy and hindcast data to develop precalculated site parameters (exceedance tables, persistence estimates, return periods, and wind and wave roses) at a similar spacing to the MHK wave atlas with temporal resolutions of one year to monthly for the U.S. West Coast, Hawaii, and Alaska.

\subsubsection{Wave Energy Prospecting Tools}

In the early stages of WEC development, when emerging technologies reach a pre-commercial stage and are marginally economic, device and project developers will likely need to identify sites that have resource characteristics that best match the design and performance of their technology, while considering other factors that impact LCOE such as distance from ports, logistical constraints, vessel costs, and energy demand. Such analyses are complicated yet are needed to help minimize project costs and maximize availability and AEP to yield the lowest LCOE. However, finding these sites is not an easy task and cannot be done using simple site characteristics such as annual average energy flux estimates and distances from shore. Thus, public tools, resources, and cost data sets are needed to help determine accurate estimates of 
project LCOE. As part of this larger LCOE tool, a module of the software should be a wave energy prospecting tool that can ingest existing wave data sets (e.g., buoy measurements, hindcast data) and automatically identify sites that have the highest AEP based on devicespecific parameters, such as the power matrix, limiting sea states, availability estimates, and O\&M scenarios. The tool could also produce maps of AEP. Such software tools could have many uses, such as allowing device developers to explore and identify sites of the highest energy production potential and to investigate the sensitivity of AEP and deployment locations on design parameters.

Gaps: No public tools are known to exist that enable WEC developers to investigate and quantify site-specific AEP based on design parameters and detailed resource conditions by month or week.

\section{Impact of Gaps:}

- Without the ability to quantify the impact of WEC design parameters on AEP and other LCOE parameters at early design stages/low TRL, the development time and cost will likely increase at higher TRLs as impacts are addressed, design changes are more difficult, and suboptimal WEC designs are likely.

- Wave characteristics and rates of occurrence are site specific and can change based on location and water depth. Without the ability to identify optimal sites for early-stage WEC deployments, margins may be reduced and project success may diminish because the best sites may not be chosen.

- Without wave-prospecting tools, device/site developers may need to develop their own prospecting tools or use time-consuming iterative methods. Either way, project costs and duration will increase.

\section{Benefits of Closing the Gaps:}

- An open-source, publicly vetted wave-prospecting tool will enable WEC developers and site developers to more easily find and filter hotspot sites for their technology. Because the tool could be open source, it could be customized by individual developers without requiring them to develop the whole framework. Such a tool would reduce project costs and allow users to quickly identify sites for development.

- An open-source tool would decrease project costs and development times and avoid duplicate work between projects.

- By providing a tool that allows device developers to determine the sensitivity of design parameters on AEP, they would be able to explore design scenarios and optimize device performance at early-design stages/lower TRLs prior to locking into a design and where designs are more quickly iterated at lower cost. 


\section{Solutions:}

- Work with technology and site developers to determine the device design parameters and metocean parameters that are important to maximize AEP and lower LCOE

- Develop a software architecture and theory that can be the basis of the wave-prospecting tool

- Code the prospecting tool and vet the methodology and tool with industry

- Implement a GIS version of the tool. 


\section{Common Themes}

The workshop identified 28 primary findings that identified gaps (problems, deficiencies, or lack of capabilities) in MHK measurement and data-processing technology. Gaps were identified for both field and laboratory measurements and processing and, while these gaps were broad in terms of impact to the MHK field, there were several themes that emerged.

\subsection{Limited Knowledge Transfer}

Over the last decade, the MHK industry has seen extensive testing activities in the laboratory and in the field. These tests have generated a wealth of experience, knowhow, and tools, yet much of this knowledge is not disseminated or is hard to find. As a result, duplicate efforts occur to develop software tools and many mistakes are repeated. While there is extensive literature within the field, it can be difficult to mine papers and reports to compile the lessons learned. There is a need for central information repositories where information and tools are globally discoverable. Through dissemination of knowledge, the MHK industry can avoid repeating mistakes, minimizing duplicate efforts, and leveraging the experience of others to help accelerate technology development and reduce costs while not compromising intellectual property.

\subsection{High Cost of Measurement}

The MHK industry is pre-commercial and has very limited budgets for testing. Unfortunately, marine grade instruments can be expensive and strain tight test budgets. Often, a tradeoff is made between the breadth and duration of a test and the number and quality of measurements. Testing is the opportunity for device developers to get information needed to understand, characterize, and advance their technology. Thus, reducing measurement can lead to incomplete data sets and reduced learning, which can have compounding impacts on technology development. By adopting mechanisms to increase instrument availability, share instrument costs between projects, increase the measurement capabilities of existing instruments, adapt instruments for MHK application, share knowledge of instrument use, and standardize measurements, measurement costs can be reduced.

\subsubsection{Better Measurement Capabilities at Low TRLs}

The TPL demonstrates that MECs can achieve rapid advancement for the lowest cost at low TRL levels (TRLs 1-3). Testing at these early stages is critical to validate numerical models and provide data on concept performance and loads. However, the measurement capabilities for smaller-scale models (1:10 and smaller) typically used at low TRLs is inadequate. The weight of sensors and DASs and the stiffness of wires connecting the device under test to external equipment can affect the device motion and performance. As well, sensors are limited or do not exist for measuring loads at small scales. For technologies to advance at smaller TRLs, new measurement technologies are needed to ensure accurate and affordable measurements.

\subsubsection{Open-Source Tools for Unified Data Processing and Analysis}

Processing and analysis of data collected during testing is the final step needed to turn the data into useful information. Most efforts in this area are performed on a project-by-project basis where custom code is developed using unique processing and visualization methods. This can lead to many factors that impact the credibility of test results, including inconsistent interpretation of data and errors in processing and calculations. Duplicate efforts between 
projects can unnecessarily increase project cost and duration. By encouraging sharing of vetted data reduction, processing, QA, and visualizations code and by adopting standard methods, the MHK industry would be able to accelerate the analysis and increase the credibility of test results. 


\section{Appendix A: Workshop Agenda}

\section{Department of Energy's $3^{\text {rd }}$ Marine and Hydrokinetic Technologies Instrumentation Workshop February 28 - March 1, 2017}

DOE's third MHK sensors and instrumentation workshop will focus on identifying measurement needs, determining gaps in measurement capabilities, and defining solution pathways for those gaps as relevant for resource assessment, laboratory and field testing, and operation of MHK technologies.

\begin{tabular}{|c|l|}
\hline Tuesday, February $\mathbf{2 8}$ \\
\hline $8: 00-8: 30$ & Registration and Continental Breakfast \\
\hline $8: 30-9: 00$ & Opening Remarks \\
\hline
\end{tabular}

\begin{tabular}{|c|c|c|}
\hline \multicolumn{2}{|c|}{ Welcome and Introduction } & Erik Mauer, DOE \\
\hline \multicolumn{2}{|c|}{ Review of Prior Workshops } & Erik Mauer, DOE \\
\hline \multicolumn{2}{|c|}{ Workshop Overview } & Rick Driscoll, NREL \\
\hline 9:00 - 10:00 & DOE Testing Instrumentation & \\
\hline \multicolumn{2}{|c|}{ MHK Instrumentation Database and Community } & Rick Driscoll, NREL \\
\hline \multicolumn{2}{|c|}{ MOIS and NWTC Component Testing } & Eric Nelson, NREL \\
\hline \multicolumn{2}{|c|}{ Tidal Turbulence Measurement System } & Levi Kilcher, NREL \\
\hline \multicolumn{2}{|c|}{ Environmental Instrumentation Workshop } & $\begin{array}{l}\text { Genevra Harker-Klimes, } \\
\text { PNNL }\end{array}$ \\
\hline \multicolumn{2}{|c|}{ MHK Activities in Canada } & $\begin{array}{l}\text { Fabian Wolk, Rockland } \\
\text { Scientific }\end{array}$ \\
\hline $10: 00-10: 20$ & \multicolumn{2}{|l|}{ Break (Coffee and Tea) } \\
\hline $10: 20-12: 00$ & \multicolumn{2}{|c|}{ State-of-the-Art in Measurement Technology } \\
\hline
\end{tabular}




\begin{tabular}{|c|c|c|}
\hline \multicolumn{3}{|c|}{ Tuesday, February 28} \\
\hline $10: 20-12: 00$ & \multicolumn{2}{|c|}{ State-of-the-Art in Measurement Technology and Testing } \\
\hline \multicolumn{2}{|c|}{ Field Testing of Wave Energy Systems } & Terry Lettenmaier, NWEI \\
\hline \multicolumn{2}{|c|}{$\begin{array}{l}\text { Field Testing of the VolturnUS 1:8 Floating Offshore } \\
\text { Wind Turbine }\end{array}$} & $\begin{array}{l}\text { Curtis Libby, University of } \\
\text { Maine }\end{array}$ \\
\hline \multicolumn{2}{|c|}{ Real-Time Wave Field Measurement and Forecasting } & $\begin{array}{l}\text { Bill Milewski, Applied Physical } \\
\text { Sciences Corp. }\end{array}$ \\
\hline \multicolumn{2}{|c|}{ Resource Assessment and Forecasting } & Robert Raye, StormGeo \\
\hline \multicolumn{2}{|c|}{ Scaled Testing of Tidal Turbines, Lab Instruments } & Martin Wosnik, UNH \\
\hline $12: 00-1: 00$ & \multicolumn{2}{|c|}{ Catered Lunch with Industry Presentations } \\
\hline \multicolumn{3}{|c|}{ Signature ADP, Kevin Frost, Nortek } \\
\hline \multicolumn{3}{|c|}{$\begin{array}{l}\text { Canada-UK projects in Turbulence Measurement and Methodology in Tidal Channels and } \\
\text { Laboratory, Fabian Wolk, Rockland Scientific }\end{array}$} \\
\hline \multicolumn{3}{|c|}{ The "Living Bridge" Tidal Energy Project, Martin Wosnik, UNH } \\
\hline 1:00-3:00 & \multicolumn{2}{|c|}{$\begin{array}{l}\text { Breakout Session } 1 \text { - Gap Identification, Characterization, and } \\
\text { Prioritization }\end{array}$} \\
\hline \multicolumn{2}{|c|}{$\begin{array}{l}\text { Findings of the Pre-Workshop Survey and Breakout } \\
\text { Group Instructions }\end{array}$} & Rick Driscoll, NREL \\
\hline \multicolumn{3}{|c|}{ Break into Groups } \\
\hline \multicolumn{2}{|c|}{ Group 1: Field Measurement: Wave } & Terry Lettenmaier \\
\hline \multicolumn{2}{|c|}{ Group 2: Field Measurement: Current } & Levi Kilcher \\
\hline \multicolumn{2}{|c|}{ Group 3: Laboratory Measurement: Wave and Current } & Eric Nelson \\
\hline $3: 00-3: 20$ & \multicolumn{2}{|l|}{ Break (Coffee and Tea) } \\
\hline $3: 20-4: 30$ & \multicolumn{2}{|l|}{ Breakout Session 1 Continued } \\
\hline $4: 30-5: 15$ & \multicolumn{2}{|c|}{ Groups Document and Prioritize Findings } \\
\hline
\end{tabular}




\begin{tabular}{|c|c|c|}
\hline \multicolumn{3}{|c|}{ Wednesday, March 1} \\
\hline $8: 00-8: 30$ & \multicolumn{2}{|l|}{ Continental Breakfast } \\
\hline $8: 30-10: 00$ & \multicolumn{2}{|c|}{ Breakout Session Reporting and Discussion } \\
\hline \multicolumn{2}{|c|}{ Group 1: Field Measurement: Wave } & Terry Lettenmaier \\
\hline \multicolumn{2}{|c|}{ Group 2: Field Measurement: Current } & Levi Kilcher \\
\hline \multicolumn{2}{|c|}{ Group 3: Laboratory Measurement: Wave and Current } & Eric Nelson \\
\hline $10: 00-10: 20$ & \multicolumn{2}{|l|}{ Break (Coffee and Tea) } \\
\hline $10: 20-12: 00$ & \multicolumn{2}{|c|}{ Breakout Session 2 - Solution Pathways } \\
\hline \multicolumn{2}{|c|}{ Group 1: Field Measurement: Wave } & Terry Lettenmaier \\
\hline \multicolumn{2}{|c|}{ Group 2: Field Measurement: Current } & Levi Kilcher \\
\hline \multicolumn{2}{|c|}{ Group 3: Laboratory Measurement: Wave and Current } & Eric Nelson \\
\hline $12: 00-1: 00$ & \multicolumn{2}{|c|}{ Catered Lunch with Industry Presentations } \\
\hline \multirow{2}{*}{\multicolumn{3}{|c|}{$\begin{array}{l}\text { TRDI ADCPs, Darryl Symonds, Teledyne RD Instruments } \\
\text { Comparison of Fault Detection Techniques for an Ocean Turbine, Pierre, FAU }\end{array}$}} \\
\hline & & \\
\hline \multicolumn{3}{|c|}{ RPS Evans-Hamilton, Trap Puckette } \\
\hline $1: 00-3: 00$ & \multicolumn{2}{|l|}{ Breakout Session 2 Continued } \\
\hline $3: 00-3: 20$ & \multicolumn{2}{|l|}{ Break (Coffee and Tea) } \\
\hline $3: 20-4: 00$ & \multicolumn{2}{|c|}{ Groups Document and Prioritize Findings } \\
\hline $4: 00-5: 30$ & \multicolumn{2}{|c|}{ Breakout Session Reporting and Discussion } \\
\hline \multicolumn{2}{|c|}{ Group 1: Field Measurement: Wave } & Terry Lettenmaier \\
\hline \multicolumn{2}{|c|}{ Group 2: Field Measurement: Current } & Levi Kilcher \\
\hline \multicolumn{2}{|c|}{ Group 3: Laboratory Measurement: Wave and Current } & Eric Nelson \\
\hline
\end{tabular}




\section{Appendix B: Pre-Workshop Survey Questions}

Question 1: In terms of MHK measurement, a) What are your top concerns or frustrations? b) What areas do you regard as satisfactory? (Please be specific and provide sufficient detail to understand context and impact.)

Question 2: How/where can MHK measurement technology be improved in the next 3-5 years to meet your data needs? (If you identify multiple improvements, please rank the improvements by impact/benefit from greatest to least.)

Question 3: What do you think the greatest technological achievement in MHK measurement technology will be in the next 5 years?

Question 4: Please include any other thoughts/suggestions/comments. 


\section{Appendix C: Workshop Participant List and Affiliations}

\begin{tabular}{|l|l|l|l|}
\hline Name & Affiliation & Name & Affiliation \\
\hline Adam Brown & $\begin{array}{l}\text { University of Washington - } \\
\text { Applied Physics Lab }\end{array}$ & Levi Kilcher & NREL \\
\hline Bill Milewski & $\begin{array}{l}\text { Applied Physical Sciences } \\
\text { Corp }\end{array}$ & Manhar Dhanak & FAU \\
\hline Bret Bosma & $\begin{array}{l}\text { O.H. Hinsdale Wave } \\
\text { Research Laboratory (OSU) }\end{array}$ & Martin Wosnik & UNH \\
\hline Bryan Murray & $\begin{array}{l}\text { CalWave Power } \\
\text { Technologies }\end{array}$ & Merrick Haller & Oregon State \\
\hline Craig Hill & U Washington & Michael Bernitsas & UMICH \\
\hline Curtis Libby & University of Maine & Michael DeLorme & Stevens University \\
\hline Darryl Symonds & TeleDyne & Patrick Cross & Hawaii \\
\hline Ean Amon & CPT & Pedro Lomonaco & Oregon State \\
\hline Eric Nelson & NREL & Pierre Beaujean & FAU \\
\hline Erik Mauer & DOE & Rick Driscoll & NREL \\
\hline Fabian Wolk & Rockland Scientific & Rick Williams & Leidos \\
\hline Gaizka Zarraonandia & DNV GL & Rob Cavagnaro & U Washington \\
\hline Genevra Harker-Klimes & PNNL & Robert Raye & StormGeo \\
\hline James Behrens & CDIP & Sam McWilliams & Integral Consulting \\
\hline James van Zweiten & FAU & Stefan Siegel & Atargis \\
\hline Johannes Spinneken & Ever Green Innovations & Tannen VanZwieten & $\begin{array}{l}\text { NASA Engineering and } \\
\text { Safety Center (NESC) }\end{array}$ \\
\hline John Sninsky & & Terry Lettenmaier & Williwaw Engineering \\
\hline Kevin Farinholt & Luna Inc & Trap Puckette & Evans Hamilton \\
\hline Kevin Frost & NortekUSA & & \\
\hline
\end{tabular}

